Portland State University

PDXScholar

\title{
Effects of Lifestyle Change and Satisfaction with Voice on Psychosocial Adjustment of Laryngectomees
}

Susan M. Nicks

Portland State University

Follow this and additional works at: https://pdxscholar.library.pdx.edu/open_access_etds

Part of the Speech and Hearing Science Commons Let us know how access to this document benefits you.

Recommended Citation

Nicks, Susan M., "Effects of Lifestyle Change and Satisfaction with Voice on Psychosocial Adjustment of Laryngectomees" (1997). Dissertations and Theses. Paper 5381.

https://doi.org/10.15760/etd.7254

This Thesis is brought to you for free and open access. It has been accepted for inclusion in Dissertations and Theses by an authorized administrator of PDXScholar. Please contact us if we can make this document more accessible: pdxscholar@pdx.edu. 


\section{THESIS APPROVAL}

The abstract and thesis of Susan M. Nicks for the Master of Science in Speech

Communication: Speech and Hearing Science were presented February 10, 1997, and accepted by the thesis committee and the department.

COMMITTEE APPROVALS:

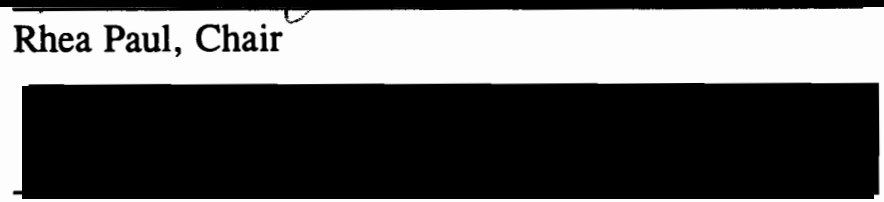

Lisa Letcher-Glembo

DEPARTMENT APPROVAL:
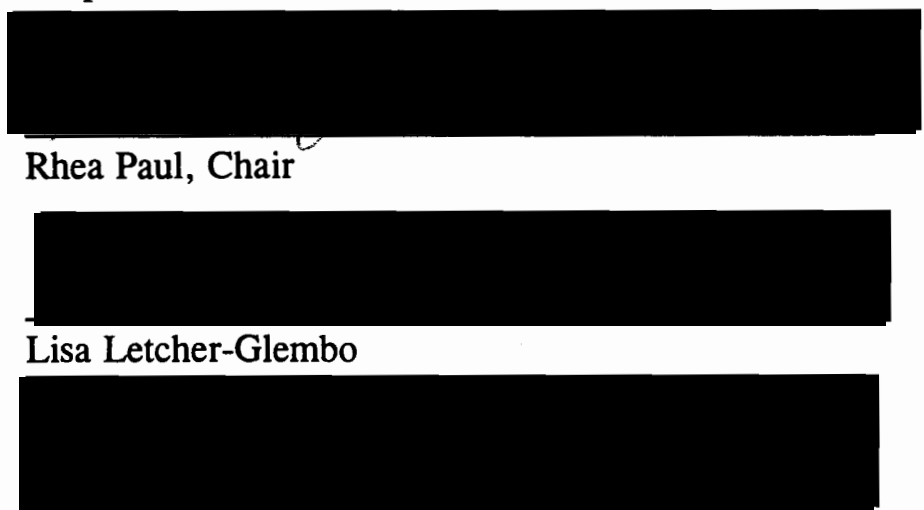

Maria Talbott

Representative of the Office of Graduate Studies

Stephen A/Kosokoff, Chair

Department of Speech Communication

$* * * * * * * * * * * * * * * * * * * * * * * * * * * * * * * * * * * * * * * * * * * * * * * * * * * * * * * * * * * * * * * * * * * * * *$

ACCEPTED FOR PORTLAND STATE UNIVERSITY BY THE LIBRARY

by on

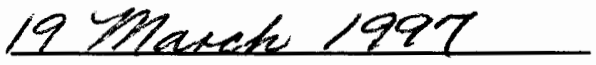




\begin{abstract}
An abstract of the thesis of Susan M. Nicks for the Master of Science in Speech

Communication: Speech and Hearing Science presented February 10, 1997.

Title: Effects of Lifestyle Change and Satisfaction with Voice on Psychosocial Adjustment of Laryngectomees.
\end{abstract}

This research sought to examine the level of psychosocial adjustment in a sample of laryngectomees by describing demographic characteristics, alcohol consumption, and tobacco use pre-cancer diagnosis and currently, satisfaction with voice, and by examining the relationship between each subject's level of psychosocial adjustment and lifestyle change, i.e. cessation of tobacco and/or alcohol consumption, and satisfaction with voice.

Twenty-one subjects completed (a) Demographic Questionnaire, (b) Lifestyle Questionnaire, (c) Satisfaction with Voice Scale, and (d) Psychosocial Adjustment to Illness Scale-Self Report (PAIS-SR). Descriptive and inferential statistics were completed.

The average age of the subject group ( 16 males, 5 females) was 72.5 years, by far the oldest group of laryngectomees studied up to this point. Average time post-onset of laryngectomy was 14 years. Ten subjects (48\%) used esophageal speech, $8(38 \%)$ used artificial larynx; and $5(24 \%)$ used tracheoesophageal devices. Eighty-six percent of the subjects reported laryngectomy support group membership. Eighty-one percent of subjects described current health status as "good" or "very good." Ninety-five percent of subjects 
reported having smoked pre-cancer diagnosis for an average of 41.25 years. Eighteen subjects $(90 \%)$ reported smoking one or more packs of cigarettes daily. Twenty subjects (95\%) reported alcohol consumption pre-cancer diagnosis, with 10 males (67\%) and 4 females $(80 \%)$ consuming alcohol daily. Self-report indicated partial lifestyle change following laryngectomy surgery. Ninety-five percent of the subjects had ceased smoking. One male subject reported one-half to one pack of cigarettes current daily consumption. Thirty-eight percent of subjects (8 males) reported current daily alcohol consumption averaging two to three units. Female subjects reported virtual cessation of alcohol consumption since laryngectomy. Results of the PAIS-SR were questionable; 5 subjects failed to answer a sufficient number of questions. Cautious analysis of the results suggests that $71 \%$ of subjects were "well-adjusted" and $29 \%$ "poorly adjusted." $T$-tests and a correlation coefficient were used to analyze the data. Significant differences were found between the scores of the "well-adjusted" group and the "poorly adjusteci group." The relationship between level of adjustment and cessation of drinking/smoking as well as the relationship between level of adjustment and degree of satisfaction with voice were not significant. 


\title{
EFFECTS OF LIFESTYLE CHANGE AND SATISFACTION WITH VOICE ON PSYCHOSOCIAL ADJUSTMENT \\ OF LARYNGECTOMEES
}

\author{
by
}

SUSAN M. NICKS

A thesis submitted in partial fulfillment of the requirements for the degree of

\author{
MASTER OF SCIENCE \\ in \\ SPEECH COMMUNICATION: \\ SPEECH AND HEARING SCIENCE
}

Portland State University

1997 


\section{ACKNOWLEDGEMENTS}

Thanks to Lisa Letcher Glembo, Rhea Paul, and Maria Talbott for their time and generous assistance to me on this thesis.

Thanks to Lisa Letcher Glembo for encouraging me to follow my bliss.

Special thanks to Dick Guarnero, Tom Day, and other volunteers from New Voice Club and the American Cancer Society. Without their participation and support, this study would not have been possible. 


\section{TABLE OF CONTENTS}

PAGE

\section{CHAPTER}

I INTRODUCTION $\ldots \ldots \ldots \ldots \ldots \ldots \ldots \ldots \ldots \ldots \ldots \ldots \ldots$

Statement of Purpose $\ldots \ldots \ldots \ldots \ldots \ldots \ldots \ldots \ldots \ldots$

Definition of Terms $\ldots \ldots \ldots \ldots \ldots \ldots \ldots \ldots \ldots$

II REVIEW OF THE LITERATURE $\ldots \ldots \ldots \ldots \ldots \ldots \ldots \ldots \ldots$

Tobacco Use and Cancer $\ldots \ldots \ldots \ldots \ldots \ldots \ldots \ldots$

Tobacco Intake Measurements . . . . . . . . . . . . . 11

Tobacco Use Following Laryngectomy $\ldots \ldots \ldots \ldots \ldots \ldots \ldots 13$

Alcohol Consumption and Cancer $\ldots \ldots \ldots \ldots \ldots \ldots \ldots 14$

Alcohol Dependency and Consumption Measurements . . . . . . . 15

Psychosocial Adjustment and Rehabilitation of

Head and Neck Cancer Patients . . . . . . . . . . . . . 19

Impact of Tobacco and Alcohol Use . . . . . . . . . . . . . . 19

Impact of Pre-operative Counseling . . . . . . . . . . 21

Impact of Type of Medical Treatment $\ldots \ldots \ldots \ldots \ldots \ldots 22$

Impact of Internal Locus of Control . . . . . . . . . . . . . 23

Impact of Premorbid Personality . . . . . . . . . . . . . 23

Impact of Overall Health Status . . . . . . . . . . . . 23

Impact of Satisfaction with Voice Quality . . . . . . . . 24

Impact of Perceived Social Support $\ldots \ldots \ldots \ldots \ldots \ldots 26$

Tools Used for Measuring Psychosocial Adjustment $\ldots \ldots \ldots \ldots 28$

Demographic Questionnaires . . . . . . . . . . . 28

Lifestyle Questionnaire . . . . . . . . . . . . . . 28

Satisfaction with Voice Scale $\ldots \ldots \ldots \ldots \ldots \ldots 28$ 
Psychosocial Adjustment to Illness Scale (PAIS) and

Psychosocial Adjustment to Illness Scale-

Self Report (PAIS-SR) . . . . . . . . . . . . . . 29

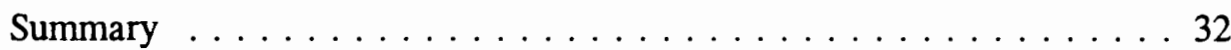

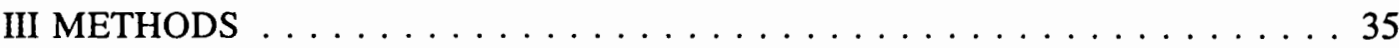

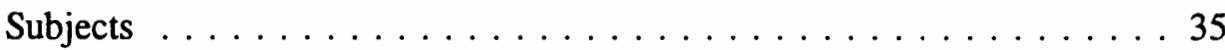

Subject Criteria . . . . . . . . . . . . . . . . 35

Subject Recruitment and Subject Consent . . . . . . . . . 36

Final Subject Distribution . . . . . . . . . . . . . . . 37

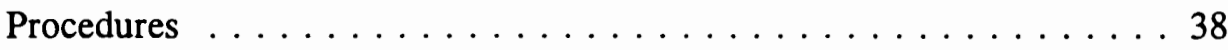

Data Collection . . . . . . . . . . . . . . . . 38

Study Measurement Tools . . . . . . . . . . . . . . . . 39

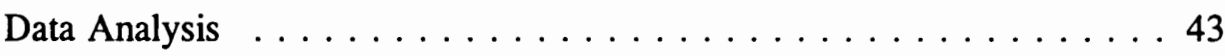

Descriptive Statistics . . . . . . . . . . . . . . . 43

Inferential Statistics $\ldots \ldots \ldots \ldots \ldots \ldots \ldots \ldots$

IV RESULTS AND DISCUSSION $\ldots \ldots \ldots \ldots \ldots \ldots \ldots \ldots \ldots$

Results ............................ . . 44

Demographic Questionnaire Results . . . . . . . . . . . . . . 45

Gender and Age Characteristics ............. 45

Time Post-Onset of Laryngectomy . . . . . . . . . . . . 45

Method of Speech Production . . . . . . . . . . . . . 46

Support Group Membership . . . . . . . . . . . . 47

Current Health Status . . . . . . . . . . . . . . 47

Lifestyle Change Questionnaire Results . . . . . . . . . . . . . . . . 47

Lifestyle Prior to Cancer Diagnosis in Regard

to Smoking Behavior . . . . . . . . . . . . . 47

Lifestyle Prior to Cancer Diagnosis in Regard

to Drinking Behavior . . . . . . . . . . . . . . . . 48

Lifestyle Following Laryngectomy in Regard to

Smoking Behavior . . . . . . . . . . . . . . . . 48

Lifestyle Following Laryngectomy in Regard to

Drinking Behavior 
Satisfaction with Voice Scale Results

Description of Current Communication Skills . . . . . . . 50

Perceived Benefits of Speech Therapy ............ 51

Self-Rating of Ability to Be Understood . . . . . . . . . 52

Psychosocial Adjustment to Illness Scale-Self Report

(PAIS-SR) Results . . . . . . . . . . . . . . . . . 52

Level of Psychosocial Adjustment and Reported Cessation of Tobacco Use and/or Alcohol Consumption . . . . . . . . . 54

Level of Psychosocial Adjustment and Degree of Satisfaction with Voice . . . . . . . . . . . . . . . . . . . 55

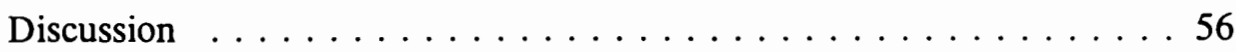

Demographic Information . . . . . . . . . . . 56

Tobacco Use and Alcohol Consumption . . . . . . . . . . 58

Psychosocial Adjustment Level and Use of the PAIS-SR

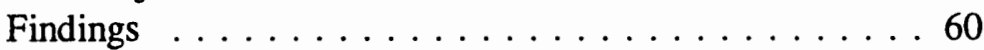

Satisfaction with Voice Scale $\ldots \ldots \ldots 63$

Study Limitations . . . . . . . . . . . . . . 63

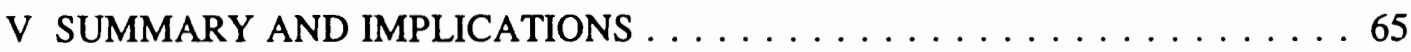

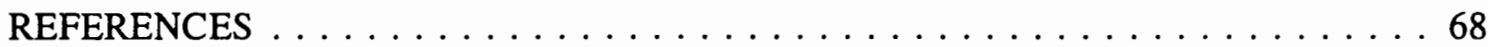

\section{APPENDICES}

A Subject Recruitment Letter $\ldots \ldots \ldots \ldots \ldots \ldots \ldots \ldots$

B Written Consent Form . . . . . . . . . . . . . . . 75

C Portland State University's Use of Human Subjects in Research

Approval Letter . . . . . . . . . . . . . . . . . 77

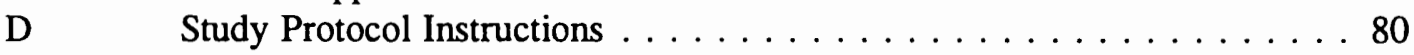

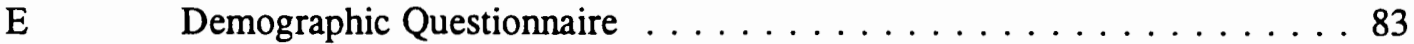

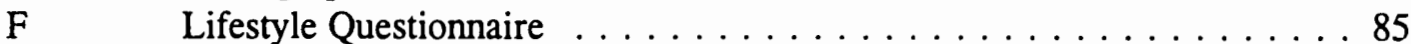

G Satisfaction with Voice Scale $\ldots \ldots \ldots \ldots \ldots$ 


\section{LIST OF TABLES}

PAGE

\section{TABLE}

1. Descriptive Statistics Grouped According to Measurement Tool . . . . . . 4

2. Psychosocial Adjustment to Illness Scale (PAIS) Internal Consistency Reliability Across Subtest Domains . . . . . . . . . . . . . . 30

3. Correlation of PAIS with Other Commonly Used Psychological Measures ..................... 31

4. Final Subject Distribution by Age and Gender $\ldots \ldots \ldots \ldots$

5. Demographic Questionnaire Results for Male Subjects, Female Subjects, and All Subjects . . . . . . . . . . . . 46

6. Lifestyle Prior to Cancer Diagnosis . . . . . . . . . . . . . . 49

7. Lifestyle Following Laryngectomy Surgery $\ldots \ldots \ldots \ldots \ldots$

8. Satisfaction with Voice Scale Results $\ldots \ldots \ldots \ldots \ldots \ldots \ldots$

9. Compilation of PAIS-SR Scores for Males, Females, and All Subjects by Averages, Range, and Category . . . . . . . . . . 53

10. Level of Adjustment as Measured by the PAIS-SR and Current Lifestyle in Regard to Tobacco Use and Alcohol Consumption . . . . . . . . . . . . . . . . . . . . . 54

11. Level of Adjustment as Measured by the PAIS-SR and Degree of Satisfaction with Voice . . . . . . . . . . . . . . 55 


\section{CHAPTER I}

\section{INTRODUCTION}

A variety of factors contribute to the psychosocial adjustment of persons who undergo laryngectomy to excise laryngeal cancer. Self-rating of voice, pre-surgical counseling, type of medical treatment, overall health status, perceived social support, and locus of control are among the variables studied in connection with the psychosocial adjustment levels of laryngectomees (Ackerstaff, Hilgers, Balm, Aaronson, \& Van Zandwijk, 1993; Blood, 1993;

Blood, Dineen, Kauffman, Raimondi, \& Simpson, 1993; Blood, Luther, \& Stemple, 1992; Blood, Simpson, Raimondi, Dineen, Kauffman, \& Stagaard, 1994; Byrne, Walsh, Farrelly, \& O’Driscoll, 1993; DeSanto, Olsen, Rohe, Perry, \& Keith 1995; Hilgers, Ackerstaff, Aaronson, Schouwenburg, \& Van Zandwijk, 1990; Johnson, Casper, \& Lesswing, 1979; Morton, Davies, Baker, Baker, \& Stell, 1984; Renner, 1995; Stam, Koopmans, \& Mathieson, 1991). The laryngectomy experience is a complex one which impacts every aspect of one's life. Although the surgery is no longer as risky as it once was (Karmody, 1988), the changes it creates are enormous. In addition to dealing with the terrifying experience of having cancer and worrying about a recurrence, the patient must deal with physical and psychological impact of loss of voice, new image, extensive self-care differences and disfigurement. Many patients fear changes in meaningful relationships and support networks. There are concerns about job loss and financial difficulty. Sometimes the patient can no longer participate in favorite activities. Since the peak incidence of laryngeal cancer is from age 60 to 79 (Spiegel \& Sataloff, 1993), the average laryngectomee also faces challenges typical to the aging 
population, including caregiving to a sick family member, widowhood, loss of family and friends, chronic or acute illness, and increasing physical disability. Successful rehabilitation is truly a challenge for all involved- the laryngectomee, his or her family, support system, and medical and rehabilitation teams.

Over the past 30 years, our knowledge concerning rehabilitation of the laryngectomee has steadily increased. For years, success or failure in attaining esophageal voice was considered the primary determinant of psychosocial adjustment. Researchers gradually came to understand that rehabilitation was a more all-inclusive concept than they had first thought; they began to explore the impact of other factors, both psychosocial and physical, on rehabilitation outcomes. There is as yet no conclusive evidence from the literature concerning which factors best predict positive psychosocial adjustment, although many of the factors under study have been found to be correlated in some way to each other, and to psychosocial adjustment. Continued investigation is necessary if communication disorders specialists are to be effective in their efforts to improve rehabilitation outcomes.

It is widely acknowledged that the etiology of head and neck cancer is related to excessive consumption of tobacco and alcohol (Hedberg, Vaughan, White, Davis, \& Thomas, 1994; Norris \& Cady, 1991; Rice \& Spiro, 1989; Stevens, Gardner, Parkin, \& Johnson, 1983). Many head and neck cancer patients have long-term problems with dependence on these substances. Abuse of tobacco and/or alcohol frequently continues even following the cancer diagnosis and laryngectomy surgery. Some members of the medical community believe that persisting dependence upon tobacco and alcohol, although difficult to measure, is one of the primary psychosocial problems of the laryngectomee following medical treatment (Breitbart \& Holland, 1988). Communication disorders specialists should be concerned about 
laryngectomees' continued use of tobacco and alcohol since substance dependence, particularly alcohol dependence, can seriously affect cognition, behavior, and communication, and therefore negatively impact overall rehabilitation and psychosocial adjustment (Breitbart \& Holland, 1988; Darvill, 1983; Perry, 1983a, 1983b).

\section{STATEMENT OF PURPOSE}

This research project, primarily descriptive in nature, had multiple purposes. The investigator sought to describe general demographic characteristics of a sample of laryngectomees, amount and frequency of alcohol consumption and tobacco use prior to cancer diagnosis and following surgery (current status), satisfaction with voice, and level of psychosocial adjustment. (See Table 1 for specific data to be reported.)

Demographic information, satisfaction with voice, and level of psychosocial adjustment are areas that have already been analyzed by other researchers, and as such, constitute familiar territory in ongoing communication disorders research about laryngectomee rehabilitation. In addition to this familiar type of investigation, data concerning tobacco and alcohol consumption were gathered. This latter type of investigation is familiar territory for medical researchers, who gather data about tobacco and alcohol consumption prior to cancer diagnosis for the purpose of analyzing variables such as risk of contracting cancer, prognostic factors for survival, and patient compliancy with treatment. Data relating to consumption of tobacco and alcohol following surgery are sparse. Thus, the primary contribution of this study is to make an initial effort toward finding what kind, if any, relationships might exist between psychosocial adjustment and consumption of tobacco and alcohol following laryngectomy. 


\section{TABLE 1}

Descriptive Statistics Grouped According to Measurement Tool

\begin{tabular}{|c|c|}
\hline $\begin{array}{l}\text { Demographic } \\
\text { Information } \\
\text { Questionnaire }\end{array}$ & $\begin{array}{l}\text { - Subject response rate } \\
\text { - Number of male subjects that participated in the study } \\
\text { - Number of female subjects that participated in the study } \\
\text { - Average age of subjects } \\
\text { - Range of age of subjects } \\
\text { - Average length of time post-onset laryngectomy surgery } \\
\text { - Range of length of time post-onset laryngectomy surgery } \\
\text { - Percentage of subjects that utilize each potential mode of speech production } \\
\text { - Percentage of subjects that report support group participation } \\
\text { - Percentage of subjects that report health status as excellent, very good, } \\
\text { good, fair or poor }\end{array}$ \\
\hline $\begin{array}{l}\text { Lifestyle } \\
\text { Questionnaire }\end{array}$ & $\begin{array}{l}\text { PART A: PRIOR-TO-CANCER DIAGNOSIS LIFESTYLE } \\
\text { - Percentage of subjects who report they smoked } \\
\text { - Average and range of number of years subjects smoked } \\
\text { - Reported amount and frequency of tobacco consumption } \\
\text { - Percentage of subjects who report consumption of alcohol } \\
\text { - Reported amount and frequency alcohol consumption } \\
\text { PART B: FOLLOWING LARYNGECTOMY/CURRENT LIFESTYLE } \\
\text { - Percentage of subjects who previously smoked that report cessation of } \\
\text { smoking } \\
\text { - Percentage of subjects who currently smoke } \\
\text { - Reported amount of current daily tobacco consumption } \\
\text { - Percentage of subjects who previously drank that report cessation of alcohol } \\
\text { intake } \\
\text { - Percentage of subjects who report current alcohol consumption } \\
\text { - Reported amount and frequency of current alcohol consumption }\end{array}$ \\
\hline $\begin{array}{l}\text { Satisfaction } \\
\text { With } \\
\text { Voice } \\
\text { Scale }\end{array}$ & $\begin{array}{l}\text { - Percentage of subjects who respond positively, negatively, or neutrally } \\
\text { when asked about self-perception of communication skills } \\
\text { - Percentage of clients who had received speech therapy } \\
\text { - For those who had received speech therapy, percentage of subjects who } \\
\text { respond positively, negatively, or neutrally when asked about their self- } \\
\text { perceptions of the benefits of speech therapy } \\
\text {-Percentage of clients who on a Likert-type } 10 \text {-point scale report being } \\
\text { "understood all the time" (Circled } 1,2 \text {, or } 3 \text { ), "understood some of the time" } \\
\text { (Circled } 4,5, \text { or } 6 \text { ), or are "rarely understood" (Circled } 7,8,9 \text {, or } 10 \text { ) }\end{array}$ \\
\hline $\begin{array}{l}\text { Psychological } \\
\text { Adjustment } \\
\text { to Illness } \\
\text { Scale - } \\
\text { Self-Report }\end{array}$ & $\begin{array}{l}\text { - classification of subjects as well-adjusted or poorly adjusted according to the } \\
\text { norms for mixed cancer patients reported in the PAIS-SR } \\
\text { - average and range of overall adjustment scores for subjects as a group } \\
\text { - average and range of overall adjustment score for male subjects } \\
\text { - average and range of overall adjustment score for female subjects }\end{array}$ \\
\hline
\end{tabular}


In this descriptive portion of the study, it was hypothesized that there would be a high percentage, $75 \%$ or more, of subjects who reported one or more of the following in relation to alcohol and tobacco consumption prior to cancer diagnosis: (a) smoked cigarettes or other tobacco product; (b) smoked 20 cigarettes (one pack) per day; (c) consumed alcohol; (d) consumed 4 or more units of alcohol per day.

For the analytical portion of the study, there were several hypotheses made:

1. It was hypothesized that there would be a significant difference between levels of adjustment for subjects who were well-adjusted versus those who were poorly adjusted.

2. It was hypothesized that higher overall satisfaction with voice would predict level of adjustment.

3. It was hypothesized that subjects who reported cessation of tobacco use following surgery would have higher levels of psychosocial adjustment than subjects who continued to use tobacco.

4. It was hypothesized that subjects who reported cessation or less than daily consumption of alcohol following surgery would have higher levels of psychosocial adjustment than subjects who consumed alcohol daily.

The null hypotheses were:

1. It was hypothesized that there would not be a significant difference between levels of adjustment for subjects who were well-adjusted versus those who were poorly adjusted.

2. It was hypothesized that higher overall satisfaction with voice would not predict level of adjustment. 
3. It was hypothesized that subjects who reported cessation of tobacco use following surgery would not have higher levels of psychosocial adjustment than subjects who continued to use tobacco.

4. It was hypothesized that subjects who reported cessation or less than daily consumption of alcohol following surgery would not have higher levels of psychosocial adjustment than subjects who consumed alcohol daily.

\section{DEFINITION OF TERMS}

Alaryngeal speech: A variety of methods of speaking without a larynx, including esophageal speech, tracheoesophageal speech, or use of an artificial larynx.

Alcohol abuse: Persistent use of alcohol in spite of negative social and physical consequences (Egbert, 1993).

Artificial larynx: A pneumatic or electronic device used to produce alaryngeal voice.

Drinker: For the purposes of this research, a person who consumes one to two units of alcohol at least one to two times per week.

Esophageal speech: Method of producing alaryngeal speech in which air is forced into the pharyngeo-esophageal segment causing it to vibrate and thereby produce sound.

Laryngectomee: A person who has had his/her larynx removed secondary to cancer in the laryngeal area.

Laryngectomy: Partial or total removal of the larynx secondary to cancer in the laryngeal area. 
Lifestyle: For the purposes of this research, lifestyle refers to smoking and/or drinking habits of a person who has experienced treatment for laryngeal cancer, including whether the person smokes and/or drinks, and the amounts consumed daily.

Lifestyle change: For the purposes of this research, lifestyle change refers to changes in smoking and/or drinking habits following diagnosis of laryngeal cancer, or medical treatment for the same.

Lifestyle Questionnaire: A questionnaire that asks the subject to quantify smoking and drinking habits prior to and following laryngectomy.

Overall satisfaction with voice: A single number score ranging from 3 to 16 , that represents a numerical combination of the three questions on Satisfaction with Voice Scale. The method for determining that number is described in the Procedures section.

Psychosocial adjustment: One aspect of rehabilitation that includes psychological and social adaptation to serious illness. Psychosocial adjustment is shaped by variables such as changes in lifestyle, chronic pain, ability to communicate, self esteem, sense of worth, depression, perception of social support, general health status, extent of surgery, etc. (Breitbart \& Holland, 1988; Mathieson, Stam, \& Scott, 1990).

Psychosocial Adjustment to Illness Scale-Self Report (PAIS-SR): A 46-question instrument developed by Derogatis and Derogatis (1990) that measures level of adaptation in seven critical areas that have relevance for adjustment to medical illness.

Rehabilitation: Overall recovery and restoration of health following serious illness; for the laryngectomy patient, rehabilitation encompasses physical recovery, social and psychological adaptation, speech and communication outcome, adaptation to disfigurement caused by surgery, and ability to function or compensate for dysfunction in all salient areas of 
one's life (Blood, 1993; Pruyn, de Jong, Bosman, van Poppel, van den Borne, Ryckman, \& de Meij, 1986).

Satisfaction with Voice Scale: A 3-question scale developed by Blood et al. (1992) for use with laryngectomees, that gathers information about how the laryngectomee perceives his/her alaryngeal speech, and the perceived quality of communication in general.

Smoker: For the purposes of this research, a person who smokes cigarettes at the rate of at least one-half pack per day.

Substance dependence:

A maladaptive pattern of substance abuse, leading to clinically significant impairment or distress, as manifested by three (or more) of the following . . . (1) tolerance ... (2) withdrawal . . . (3) the substance is often taken in larger amounts or over a longer period than was intended, (4) there is a persistent desire or unsuccessful efforts to cut down or control substance use, (5) a great deal of time is spent in activities necessary to obtain the substance . . . (6) important social, occupational, or recreational activities are given up or reduced because of substance use, (7) the substance use is continued despite knowledge of having a persistent or recurrent physical or psychological problem that is likely to have been caused or exacerbated by the substance. (American Psychiatric Association, 1994)

Tracheoesophageal speech: Type of alaryngeal speech that involves surgical creation of a fistula from the trachea into the esophagus and insertion of a one-way valve that vibrates and thereby creates sound.

Unit of alcohol: For the purposes of this study, one unit equals one shot of hard liquor, four ounces of wine, or eight ounces of beer. 


\section{CHAPTER II}

\section{REVIEW OF THE LITERATURE}

Laryngectomees' experiences with surgery and its physical, functional, psychological, and social outcomes can be devastating. The surgery itself results in disfigurement of the neck, loss of voice, and diminishment of the senses of smell and taste. Because we rely heavily on the head and neck region to show our emotions and to interact socially, disfigurement in this area has a more serious psychological effect than disfigurement of other areas of the body; these are losses which the laryngectomee cannot hide or disguise (Breitbart \& Holland, 1988; David \& Barritt, 1982; Nahum \& Golden, 1963; Weber \& Reimer, 1993). The permanent loss of normal voice is widely considered to be the most debilitating aspect of total laryngectomy (Gates, Ryan, Cooper, Lawlis, Cantu, Hayashi, Lauder, Welch, \& Hearne, 1982; Mathieson, Stam, \& Scott, 1990; Salmon, 1986; Shapiro \& Kornfeld, 1987). Many authors enumerate the common emotional responses to laryngectomy surgery and the changes that laryngectomees are forced to make in their lives: depression, anger, frustration, anxiety, nervousness, denial, self-pity, grief, inadequacy, isolation, apathy, irritability, embarrassment, shame, uncertainty about the future, and loss of self esteem (Blood, 1993; Blood et al., 1992; Gates et al., 1982a; Johnson et al., 1979; Maas, 1991; Pruyn et al., 1986; Salmon, 1986; Weber \& Reimer, 1993). In addition to these negative emotions, many laryngectomees have reported changes for the worse in their social relationships: avoiding friends, reducing social activities, increased family arguments, increased irritable behavior, marital distress, and in some cases, alcoholism or suicide (Barton, 1965; Blood, 1993; Maas, 
1991). Both patients and their families have a difficult time adjusting to the physical, psychological, and social changes which come as a result of surgery (Johnson et al., 1979). Adjustment issues can be complicated by tobacco and alcohol dependency. Heavy usage of tobacco and alcohol is the most common etiology of head and neck cancer. Smoking increases risk of cancer 5.8 times; alcohol increases it 3.6 times; smoking and alcohol increase risk 19 times; both have dose-related risk effects (Hedberg, Vaughan, White, Davis, \& Thomas, 1994; Stevens et al., 1983). Although some patients are found never to have smoked or drank alcohol, the usual scenario is one of tobacco and alcohol dependency which seems to be more common in head and neck cancer patients than in other cancer patients (Breitbart \& Holland, 1988; Perry, 1983a; Shapiro \& Kornfeld, 1987). The following is a discussion of the tobacco and alcohol consumption in the head and neck cancer population, and some of the physical, psychological, and social factors that have been suggested to affect the rehabilitation of laryngectomy patients.

\section{TOBACCO USE AND CANCER}

Compelling statistics show that a large percentage of head and neck cancer patients smoke. King, Fowlks, and Pierson (1968), in their study of 62 veterans who were laryngectomy patients, reported that $98 \%$ of their patients were smokers. Himbury and West's (1985) study of 171 laryngectomy patients showed that $76 \%$ smoked at the time of having to seek help for problems in their throats, and an additional $15 \%$ had been smokers, but had quit prior to seeking help. Even after being diagnosed with laryngeal cancer, $42 \%$ of the 171 patients continued smoking right up to the time of their surgery. Pradier, Gonzalez, Matos, Loria, Adan, Saco, and Califano (1993) did a retrospective study on 296 laryngeal cancer 
patients who had undergone radical treatment. Their pre-surgery smoking habits were as follows: $5 \%$ had been non-smokers; $9 \%$ had smoked less than 20 cigarettes per day; $50 \%$ had smoked $20-39$ cigarettes per day; $26 \%$ had smoked 40 or more cigarettes per day; there was no data available on the remaining 10\%. De Maddalena and Pfrang (1993) in Germany asked 62 male patients with laryngeal or pharyngeal malignancies about their smoking habits; $80 \%$ of them reported smoking.

Head and neck cancer patients smoke at a higher rate than the general population. Two case-control studies were done in Germany, the first with 200 male head and neck cancer patients, and the second with 164 male laryngeal cancer patients. In the first study, the $95.5 \%$ of the cancer group were smokers, and $70.5 \%$ of the controls were smokers. In the second study, $95.8 \%$ of the cancer group were smokers, and $71.5 \%$ of the control group were smokers (Maier, Dietz, Gewelke, Heller, \& Weidauer, 1992). A case control study done in western Washington by Hedberg et al. (1994) showed that $57 \%$ of controls had never smoked, or had quit more than 15 years earlier, whereas only $13.6 \%$ of laryngeal cancer cases fir these qualifications.

Interestingly, fewer women than men who were diagnosed with head and neck cancer smoke cigarettes. A retrospective study in France compared 2,539 male patients with 316 female patients who had been treated for cancer of the mouth, pharynx, or larynx. Ninety-eight percent of male patients indicated that they had smoked, but only $61.4 \%$ of female patients had smoked (Luce, Guenel, Brugere, Leclerc, Point, \& Rodriguez, 1988). The authors noted that the age at which cancer occurred in the non-smokers was significantly later than in those who smoked. 


\section{TOBACCO INTAKE MEASUREMENTS}

Patients who have cancer of the oral cavity, pharynx, or larynx are even more likely to be heavy smokers than those with other types of head and neck cancer. Barton (1965) noted that $98 \%$ of his 50 head and neck cancer patients smoked one or more packs of cigarettes a day. Stevens et al. (1983) measured tobacco and alcohol usage in a study group of 200 patients with cancer of the oral cavity, pharynx, or larynx, and in a control group of 69 patients with other head and neck cancers which are not associated with tobacco or alcohol usage. Before diagnosis, $84 \%$ of the study group smoked, compared with $32 \%$ of the control group. In the study group, $9.5 \%$ smoked one-half pack per day, $40 \%$ smoked one pack per day, and $24 \%$ smoked two or more packs per day. Three and a half percent smoked cigars or pipes, and $7 \%$ were past smokers who quit. In the control group, $7 \%$ smoked one-half pack per day, $12 \%$ smoked one pack per day, and $4 \%$ smoked two or more packs per day. Three percent smoked pipe or cigar, and $6 \%$ were past smokers who had quit. Following diagnosis, $11 \%$ of the study group continued to smoke one-half pack per day, $17 \%$ smoked one pack per day, $8.5 \%$ smoked two or more packs per day, and $1.5 \%$ smoked pipes or cigars. Over half the smokers quit smoking. Finally, Ackerstaff, Hilgers, Aaronson, and Balm (1994) in the Netherlands said that of their 63 head and neck cancer patients, $98 \%$ smoked, and the average consumption was 22 cigarettes per day.

Laryngeal cancer patients smoke at a significantly higher rate than the general population, and they smoke significantly more than persons in the general population who do smoke. In the pair of case control studies mentioned above (Maier et al., 1992), mean tobacco consumption for head and neck cancer patients was about two times that of controls $(p<$ 0.0001). (It was not indicated which statistical methods were performed.) A clear dose- 
response relationship was found between risk for cancer and "tobacco years" (a single number showing amount and number of years tobacco was consumed). The authors calculated that the risk of laryngeal cancer increased 12 -fold for heavy smokers $(p<0.0001)$.

\section{TOBACCO USE FOLLOWING LARYNGECTOMY}

While some individuals continue to smoke following cancer diagnosis but prior to surgery, most head and neck cancer patients quit smoking following surgical intervention. Those who do quit have a decreased risk of cancer recurrence (Stevens et al., 1983). Still, a surprising number of these patients try to take up smoking again. De Maddalena and Pfrang (1993) found that even when patients believed that tobacco consumption had been the cause of their cancer, there was no correlation of this belief with cessation of smoking following surgery.

In Himbury and West's (1985) survey, $40 \%$ of the 130 smokers tried smoking after surgery; $19 \%$ of them kept smoking, however, at a much reduced average of 10 cigarettes per day. In Kommers and Sullivan's (1979) questionnaire study, 45 wives of laryngectomees reported that $13.3 \%$ of their husbands continued to smoke. Of Wallen and Webb's (1975) 100 male laryngectomy patients, $77 \%$ indicated that they no longer smoked, and $3 \%$ said that they continued to smoke one or more packs per day. Of the 100 female laryngectomy patients, $84 \%$ indicated that they no longer smoked, and $2 \%$ said that they continued to smoke one or more packs per day. Finally, in the Ackerstaff et al., (1994) study, $9 \%$ of the 62 smokers continued smoking following total laryngectomy.

Patients who undergo total laryngectomies become unable to inhale through the mouth to the lungs. This means that their addiction to nicotine can no longer be satisfied by smoking 
in the conventional way (through the mouth). Himbury and West (1985) asked the 27 patients who had stopped smoking following laryngectomy why they had done so: $59 \%$ said that smoking had lost its satisfaction; $21 \%$ said that they wanted to avoid further health risks; $7 \%$ had been told to give up smoking; the remaining $9 \%$ gave various other reasons.

\section{ALCOHOL CONSUMPTION AND CANCER}

Alcohol as a risk factor for head and neck cancer is not as clearly understood as tobacco (Breitbart \& Holland, 1988; Freudenheim et al., 1992; Maier et al., 1992). Alcohol may be a chemical carcinogen, in which case it is a direct initiator of cancer; it may be a co-carcinogen, which means it potentiates carcinogenic agents such as tobacco; also, it may be a promoter of tumor growth (Breeden, 1984; Maier et al., 1992). Although the role of alcohol remains unclear, the risk created by consumption of both cigarettes and alcohol is greatly increased. Cigarette smoking alone increases risk of laryngeal cancer 5.8 times, alcohol consumption alone increases risk 3.6 times, but consumption of both cigarettes and alcohol increases risk 19 times (Stevens et al., 1983); this is called a synergistic effect, in which the combined risk is higher than simply adding the two risk factors together. In the Stevens et al. (1983) study, it was found that those patients who continued smoking after diagnosis were at increased risk for recurrence of cancer, but there was not a clear relationship between continued alcohol consumption and recurrence. The authors believed that alcohol has a promotion effect when combined with tobacco.

Alcohol can interfere with medical treatment and actually create toxicity, for example, when it interacts with radiation therapy (Breeden, 1984). Thus, it is not surprising that patients who continue drinking after surgery have an increased likelihood of complications which 
interfere with their follow-up treatment, rehabilitation, and healing process, not to mention the increased risk of a second primary cancer at a site near the original one (Perry, 1983a; Breeden, 1984; King et al., 1968; Lowry, 1975; Breitbart \& Holland, 1988). Both Breeden (1984) and Lowry (1975) believe that a history of alcohol dependency can change decisions about primary care and that it is necessary to treat the alcohol problem aggressively.

\section{ALCOHOL DEPENDENCY AND CONSUMPTION MEASUREMENTS}

Discovering whether a patient is alcohol-dependent is not always a straightforward task. It is possible that the issue of whether and how much patients drink has not been wellinvestigated due to the unwillingness of subjects to answer questions about their drinking habits. Lowry (1975) indicated that most alcoholic patients are able to stay sober long enough to visit a doctor, and thus are able to hide their drinking problem. He mentions a survey he did in 1970 in which fully one-third of the patients were "obviously" alcoholic; he believed that if he had investigated more fully, the incidence would have been found to be "much higher" (p. 1276). In the study described in the same article, 100 consecutive head and neck patients were asked about drinking habits. Many of the patients tried to hide their drinking problem, so Lowry sought information from relatives or friends of the patients. The incidence of alcoholism $(60 \%)$ was found to be twice as high as originally estimated with this population. Finally, doctors are often hesitant to diagnose alcoholism because it can be "interpreted as a moral judgment" (Lowry, 1975, p. 1276). The result is likely to be under-diagnosis. Lowry's efforts to find out the incidence of alcoholism in his head and neck patients only served to confirm his belief in the difficulty of diagnosis. 
Finding out about a patient's alcohol consumption before or after medical treatment can also be difficult due to habits of denial which are typical of the alcoholic personality (King et al., 1968; Lowry, 1975). Efforts to find out about alcohol usage are easily thwarted by silence on the part of the alcoholic. Gates et al. (1982a) studied 53 laryngeal cancer subjects prior to their hospitalization, and 40 laryngectomees following surgery. Questions about smoking were answered readily enough by all the patients, but when it came to being candid about drinking habits, $43 \%$ of the first group and $83 \%$ of the second group did not respond to those questionnaire items related to alcohol consumption.

Although several studies have sought to quantify the incidence of alcoholism in head and neck cancer patients, early studies give primarily estimates. King et al. (1968) believed that the incidence of alcoholism in their laryngeal cancer patients was "significantly high" (p. 195 ) - they estimate $25-50 \%$. Barton (1965) estimated that $24 \%$ of his 50 patients were alcoholic. In Lowry's (1975) study, patients with supraglottic cancer were considered to have a moderate incidence of alcoholism at $57 \%$. Vocal chord cancer had a low incidence of alcoholism at 7\%. De Maddalena and Pfrang (1993) indicated that $83 \%$ of their patients drank daily, and more than $50 \%$ could be "considered" alcoholic.

More recent studies have tried to distinguish between light, moderate, and heavy drinking. Pradier et al. (1993) found that $18 \%$ percent of their subjects with laryngeal cancer were non-drinkers, $26 \%$ were moderate drinkers, $44 \%$ were heavy drinkers $(75 \mathrm{ml}$ or more per day), and there was no data about $11 \%$. In their study, amount of alcohol intake was found to have a statistically significant relationship to prognosis for survival in 296 male patients treated for laryngeal cancer. 
As with smoking, women who contract head and neck cancer are found to drink at lower rates than men. Luce et al. (1988) found that the percentage of drinkers (in their sample of 2,539 men and 316 women) was significantly higher in men than in women ( $96 \%$ versus 63.3\%); "drinker" was not defined.

Diagnosed head and neck cancer patients have been found to drink or abstain from drinking at approximately the same rate as the general population. Eighty-nine percent of the head and neck cancer subjects in the Maier et al. (1992) study were alcohol consumers compared to $88 \%$ of controls (persons from the general population). It is noted, however, that for those head and neck cancer patients who do drink, amount of intake is significantly higher. The intake of the cancer subjects was an average of 69 grams/day versus 29 grams/day for the general population. As for the subjects with laryngeal cancer, their intake was also higher than that of controls (65 grams/day versus 29 grams/day). The results of a case control study done by Hedberg et al. (1994) are consistent with Maier and colleagues' findings. Alcohol intake measured in drinks per week was found to be significantly higher for laryngeal cancer patients than for the general population. While $76 \%$ of controls reported fewer than 7 drinks per week, only $41 \%$ of cases did. Twelve percent of controls and $19 \%$ of cases reported 7 to 13 drinks per week; $5 \%$ of controls and $12 \%$ of cases reported 14 to 20 drinks per week; $5 \%$ of controls and $17 \%$ of cases reported 21 to 41 drinks per week; and finally, $2 \%$ of controls and $11 \%$ of cases reported more than 42 drinks per week.

Above, subjects with laryngeal cancer in the Maier et al. (1992) study were found to have slightly lower intake than other head and neck cancer patients. In Stevens and colleagues' (1983) study, patients were divided into two groups: a control group of non-oral, non-laryngeal head and neck cancer, and a study group of 200 patients who had been 
diagnosed with cancer of the oral cavity, pharynx, or larynx. The study group was found to drink at higher rates than the control group. Alcohol intake was measured in units per day. One unit equaled $30 \mathrm{ml}$ (one shot) of whiskey, $120 \mathrm{ml}$ (4 ounces) of wine, or $240 \mathrm{ml}$ (8 ounces) of beer. Those who drank occasionally to one unit per day were considered light drinkers; consumers of two to three units per day were moderate drinkers; consumers of four or more units per day were heavy drinkers. The authors describe $73.5 \%$ of the study group and $24 \%$ of the control group as drinking before diagnosis. Within the $73.5 \%, 20.5 \%$ of the study group was classified as light, $19.5 \%$ as moderate, and $27.5 \%$ as heavy drinkers. Similarly, $20 \%$ of the control group were found to be light drinkers, but only $4 \%$ were classified as heavy drinkers. Following diagnosis, only $33 \%$ of the drinkers in the study group quit drinking. The authors did not find a significant relationship between cancer recurrence and continued alcohol use following cancer diagnosis.

A larger percentage of patients with laryngeal cancer are likely to be alcoholdependent than persons in the general population. In their case control study of 235 diagnosed laryngeal cancer patients and 547 controls from the general population, Hedberg et al. (1993) used the Michigan Alcoholism and Screening Test (MAST) to estimate percentages of persons who were unlikely to be alcoholic, possibly alcoholic, and probably alcoholic. Thirty-three percent of laryngeal subjects and $66 \%$ of controls were classified as unlikely to be alcoholic; $26 \%$ of laryngeal subjects and $22 \%$ of controls were classified as possibly alcoholic; $40 \%$ of laryngeal subjects and $12 \%$ of controls were classified as probably alcoholic. The strongest predictor of MAST score was the number of drinks per week. Interestingly, those subjects who smoked $20+$ cigarettes per day were more likely to be alcoholic than those who never smoked, and men were more likely than women to be alcoholic. 


\section{PSYCHOSOCIAL ADJUSTMENT AND REHABILITATION OF HEAD AND NECK CANCER PATIENTS}

Cancer survivors are affected by many psychosocial factors. Psychosocial adjustment refers to one aspect of rehabilitation that includes the psychological and social adaptation to serious illness (Breitbart \& Holland, 1988) It is an important component of recovery from cancer (Blood et al., 1992; Gates et al., 1982a). In early research efforts, investigation of post-laryngectomy communication disorders focused on psychosocial variables which related to speech rehabilitation only, but gradually, investigators have expanded their studies to explore the broader perspective of general psychological adjustment (Stam et al., 1991). Some of the factors that affect psychosocial adjustment to laryngectomy are discussed below.

\section{Impact of Tobacco and Alcohol Use}

Because tobacco plays such a major role in causation of head and neck cancer, and because many patients try to resume smoking following surgery, it would be of great interest to investigate what effect continuation or cessation of smoking has upon psychosocial adjustment, if any. Conventional wisdom says that persons who quit smoking have problems with irritability and moodiness. Recent laryngectomees certainly have their share of problems without having to cope with tobacco withdrawal symptoms. De Maddalena and Pfrang (1993) are the only authors who thus far have examined the impact of tobacco use on psychosocial adjustment of patients following surgery. They were unable to find any correlation between cessation of smoking and psychosocial adjustment.

As with smoking, de Maddalena and Pfrang's (1993) is the only study that tries to measure psychosocial adjustment in relation to lifestyle change in terms of reduction of alcohol intake. The patients who considered drinking to be a significant cause of their cancer 
tended to reduce their alcohol intake, but experienced increased psychological stress rofíowing medical treatment.

It would be of great interest to find out whether laryngectomees stop drinking alcohol following surgery, and what effect it has on their psychosocial adjustment. Does quitting drinking facilitate psychosocial adjustment, or as de Maddalena and Pfrang (1993) suggest, do laryngectomees become more susceptible to stress if they quit drinking?

Alcohol consumption can affect medical treatment as well as the rehabilitative efforts of the communication disorders specialist. Heavy alcohol use influences the laryngectomee's ability to understand what is happening to him/her, and to learn and adjust to a new life situation; there may be problems retaining information, cooperating with treatment and selfcare, concentrating on what is being said, and relating to others (Darvill, 1983; Gilmore, 1986; Perry, 1983b; King et al., 1968; Breitbart \& Holland, 1988). Alcohol abuse has a significant impact on the family. There may be a history of marital problems secondary to alcohol dependency; the alcoholic may have a poor social support network; performance at work may be compromised by alcohol dependence; there may be financial instability (Breitbart \& Holland, 1988). In addition, Lowry (1975) pointed out that all patients who had been staged at $\mathrm{T} 3$ or $\mathrm{T} 4$, many of whom had delayed having their cancers diagnosed, were alcoholic. The "T" parameter in cancer staging represents tumor depth and extent of mucosal spread. TIS, or in situ tumors, are the most superficial; T1 means that the carcinoma is confined to the region in question; $\mathrm{T} 2$ means that the carcinoma has extended to a neighboring region; T3 tumors have resulted in fixation of the vocal folds, and T4 includes connective tissue spread. A tumor that reaches T4 stage is massive and has spread beyond the larynx or has destroyed cartilage (Maisel \& Cohen, 1988). Tumors staged at T1 or T2 can usually be 
treated with radiation or partial laryngectomy; in this case, the patient is able to attain nearnormal voice and swallowing function (Logemann, 1983; Fried, 1988). However, tumors staged at $\mathrm{T} 3$ or $\mathrm{T} 4$ usually require a total laryngectomy, and these later-staged cancers carry a poorer prognosis than cancers that have been discovered at earlier stages; the normally expected cure rate is cut in half (Fried, 1988; Norris \& Cady, 1991; Spiegel \& Sataloff, 1993) All of these factors result in a poorer prognosis for the rehabilitation of those laryngectomy patients who are heavy users of alcohol, and increases the likelihood of a lengthy period of rehabilitation (Byrne et al., 1993; Darvill, 1983). Although Darvill (1983) indicated that a significant number of laryngectomees remain dependent upon alcohol following surgery, there is actually limited research available to support this statement.

Breitbart and Holland (1988) believe that individuals who are tobacco- and alcoholdependent before developing head and neck cancer demonstrate the personality characteristics of "dependence, inability to change habits, and poor adaptive coping skills" (p. 64), and that these characteristics make head and neck cancer patients less able to cope with all that their illness entails.

\section{Impact of Pre-operative Counseling}

A survey done by Johnson et al. (1979) concluded that providing more pre-operative information, counseling by a communication disorders specialist, and a visit from a fellow laryngectomee facilitate psychological adjustment in patients. These procedures are now routine occurrences in most hospitals. Stam et al. (1991) similarly found that laryngectomees who were better adjusted had received pre-operative counseling and had a brief hospital stay. 


\section{Impact of Type of Medical Treatment}

Researchers do not agree on whether psychosocial adjustment is correlated with type of treatment. In a study by Morton et al. (1984), subjects who had been treated for head and neck cancer were divided into groups according to type of treatment: surgery, radiation, or salvage surgery following failed radiation. The researchers expected to find differences in psychological well-being and life satisfaction based on the type of treatment received, but found none. Those patients who had scored poorly on measures of well-being indicated that their psychological distress was a result of pain and reduced social contacts. However, in Byrne and colleagues' (1993) pilot study of depression following laryngectomy, type of treatment was found to make a difference in the presence or absence of depression. Ten laryngectomized patients were matched with a control group of 10 patients who had received radiation therapy only for laryngeal cancer (therefore, the cancer was less invasive than that of patients who had to undergo laryngectomy). No one in the control group experienced depression, but 4 out of the 10 laryngectomy patients were depressed as measured by the Hamilton Rating Scale for Depression and the Beck Depression Inventory.

Type of surgery may make a difference in the sense that those patients who undergo a partial laryngectomy and have no permanent stoma show better adjustment than patients who undergo a near-total or total laryngectomy. DeSanto et al. (1995) suggest that the presence of a permanent stoma may have more impact than what was previously believed. In their study of 111 patients with total laryngectomies, 37 patients with near-total laryngectomies, and 23 patients with partial laryngectomies, the authors administered the Psychosocial Adjustment to Illness Scale (PAIS) and the Mayo Clinic Post-Laryngectomy Questionnaire (MCPLQ). The total and near-total patients showed similar levels of psychosocial adjustment, both lower than 
the levels of psychosocial adjustment of patients with partial laryngectomies, who had no permanent stoma.

\section{Impact of Internal Locus of Control}

Cancer researchers have shown that internal locus of control, or the belief that one controls one's own health and behavior, is correlated with positive adjustment and health outcomes. For laryngectomees, the prospect of loss of voice intensifies the issue of locus of control (Renner, 1995). Blood et al. (1993) assessed locus of control in 63 laryngectomees. Those who reported internal locus of control had better psychosocial adjustment and fewer communication problems.

\section{Impact of Premorbid Personality}

Premorbid personality and maturity have an effect on ability to adapt to the changes that accompany laryngectomy (Breitbart \& Holland, 1988). Gates et al. (1982b) suggest that flexibility, imagination, independence, and self-assuredness are important personality traits which facilitate adjustment to new circumstances. However, the very individuals who are at risk for developing laryngeal cancer are those who have abused alcohol; they are likely to have personality characteristics such as dependence, poor adaptability, denial, and difficulty changing habits. These characteristics do not lend themselves to positive psychosocial outcomes (Breitbart \& Holland, 1988; Shapiro \& Kornfeld, 1987).

\section{Impact of Overall Health Status}

Problems with physical functioning can have an effect on psychosocial adjustment. Hilgers et al. (1990) found that respiratory problems which are common during the first year or two following surgery have an effect on daily living and psychological state. The problems 
include sputum production, coughing, nasal discharge, stoma cleaning, forced expectoration, breathlessness, and wheezing. Their effect on daily living was to cause sleep problems, fatigue, and poor voice quality leading to anxiety, depression, and fewer social contacts. When laryngectomees used a heat and moisture exchanger to improve respiratory function over a 3-month period, their fatigue and sleep problems decreased, their voice quality improved, anxiety and distress decreased, and social contacts increased (Ackerstaff et al., 1993).

Impact of Satisfaction with Voice Quality

A constellation of factors that seem to be consistently correlated with positive psychosocial adjustment and with each other are: high self-esteem, low stress, positive perception of one's own voice quality, and satisfaction with social support network. Conversely, poor self-esteem, high stress, and dissatisfaction with one's own voice quality lend themselves to poorer psychosocial adjustment.

Positive perceptions of oneself and one's voice seem to have high correlations with psychosocial adjustment. Blood et al. (1992) studied 41 total laryngectomees to measure coping and adjustment. He did so by dividing the subjects' data according to type of alaryngeal speech utilized and length of time post-onset. Instruments used included a reading of The Rainbow Passage, face-to-face interviews, the Self-Esteem Scale, the Affect Balance Scale, the Psychosocial Adjustment to Illness Scale (PAIS), and the Ways of Coping Checklist-Revised (WCCL-R). The face-to-face interview included questions about: (a) laryngectomees' perceptions of their communication skills; (b) whether speech therapy had helped them adjust to or cope with cancer; (c) laryngectomees' perceptions of their ability to be understood by others on a 1 to 10 scale. 
The overall results of this study showed that $73 \%$ of the subjects had positive psychosocial adjustment and $27 \%$ had poor adjustment as determined by the PAIS. As for self-rating of voice and communication skills, $68 \%$ of the subjects felt they were communicating "just fine," $15 \%$ said they were communicating "poorly," and 17\% were unsure of how they were communicating. Sixty-six percent of subjects believed that speech therapy had helped them in their adjustment process, $10 \%$ said it had not helped, and $24 \%$ were unsure. Seventy-three percent of the subjects rated themselves as "very good" speakers.

Type of alaryngeal speech was not found to have any relationship to how welladjusted the laryngectomees were, but length of time post-onset did. The same statistics above were categorized in terms of time post-onset with some revealing results. Those patients who were more distant from the diagnosis in time had better coping, adjustment, and overall sense of well-being; $60 \%$ of recent survivors ( 9 to 36 months post-onset) and $84 \%$ of distant survivors (40 to 98 months) had positive adjustment scores. Distant survivors tended to rate their communication skills more highly than recent survivors; $79 \%$ said they were communicating "just fine" in contrast to $31 \%$ of recent survivors who said "just fine." Eighty percent of distant survivors indicated that speech therapy had been helpful in adjusting to cancer, in contrast to $38 \%$ of recent survivors; and finally, $80 \%$ of distant survivors rated themselves as good speakers, in contrast to $44 \%$ of recent survivors.

Conversely, laryngectomees who report poor self-esteem and high stress tend to be dissatisfied with their voices. Blood (1993), in the process of developing and assessing a questionnaire which deals with communication needs of laryngectomees, Self-Evaluation of Communication Experiences After Laryngectomy (SECEL), administered a series of standardized tests to measure stress, response to the experience of having cancer, and 
subjective opinion of one's own voice. The measures included the PAIS-SR, The Impact of Event Scale (IES), The Global Assessment of Recent Stress (GARS), The Self-Esteem Scale (SES), and an author-created measure called Self-Acceptability of Voice Rating (SAVR). In the latter measure, Blood asks each subject to rate his or her own voice quality and intelligibility on a scale of 1 to 7 .

Overall results showed that of 102 subjects, $77 \%$ had positive psychosocial adjustment and $23 \%$ had poor adjustment as measured by the PAIS. These results seem to be consistent with those of his earlier study. Subjects who had negative perceptions of their voices also had low self-esteem and greater stress in their lives than did patients with positive perceptions of their voices. These subjects received high scores on the SECEL, indicating that they had not adjusted well to their new voice.

\section{Impact of Perceived Social Support}

In a later study by Blood et al. (1994), self-perception of voice quality had a high correlation with perceived social support and psychosocial adjustment. This time, 90 laryngectomees took the Psychosocial Adjustment to Illness Scale-Self Report (PAIS-SR), and according to the results, were divided into groups of "good copers" (70\%) and "poorer copers" $(30 \%)$. All the subjects then were administered three social support measures and one voice satisfaction measure: The Norbeck Social Support Questionnaire (NSSQ), the Duke University-University of North Carolina Functional Social Support Questionnaire (DUFSS), The Lubben Social Network Scale (LSNS), and Satisfaction with Voice Scale, which had been developed by the authors. The Satisfaction with Voice Scale consisted of three questions: (a) "Describe how you think you are communicating"; (b) "How did speech therapy help with adjusting or coping with the cancer?"; (c) "On a scale of 1 to 10 , how would you rate your 
ability to be understood; I mean, do people understand you?" $(1=$ rarely understood; $10=$ understood all the time). Results of this self-rating were as follows: On question $1,78 \%$ of good copers and $41 \%$ of poorer copers believed that they were communicating effectively, while $8 \%$ of good copers and $41 \%$ of poorer copers believed that they were not communicating well. On question $2,89 \%$ of good copers and $30 \%$ of poorer copers felt that speech therapy had been helpful, while $5 \%$ of good copers and $30 \%$ of poorer copers felt that it had not been helpful. On question 3, good copers gave themselves an average score of 7.2 on speaking skill, and poor copers gave themselves an average of 4.3. The relationship among the support measures and the voice measure was determined, and the authors found that good copers had a positive opinion of their new voice, and believed that they had adequate social support networks. Conversely, poorer copers had a negative opinion of their new voice and were unsatisfied with their social support network.

Stam et al. (1991) also found satisfaction with social support network to be a significant predictor of psychological adjustment following surgery. They did a very thorough assessment of 51 laryngectomees in Alberta, Canada, in which they measured demographics, current health variables, social network and support, counseling which had been provided, change of lifestyle, and quality of life. The Social Network Index (SNI) and the Social Support Questionnaire (SSQ) were used to find out type and extent of social network and satisfaction with it. Satisfaction with social support was found to be predictive of low levels of distress and higher quality of life, much more so than actual extent of social network. 


\section{TOOLS USED FOR MEASURING PSYCHOSOCIAL ADJUSTMENT}

Psychosocial adjustment can impact rehabilitative outcomes. As a result, tools have been developed to assist in measuring psychosocial outcome. These tools primarily target factors thought to affect psychosocial status. Tools pertinent to this study are discussed below.

\section{Demographic Questionnaires}

This type of questionnaire varies from study to study. It usually includes basic information such as gender and age, but can also include occupation, level of education, marital status, income, native language, race, type of communication used, number of persons living with the laryngectomee, length of time spent post-operatively in the hospital, and length of time post-surgery.

\section{Lifestyle Questionnaire}

This refers specifically to a questionnaire used by Stevens et al. (1983) in which patients with head and neck cancer answered questions about whether they had smoked tobacco and drunk alcohol both before and after their cancer diagnosis, what kind of tobacco and alcohol they had consumed, and how much of each. The information was used to discover whether continued consumption of tobacco and alcohol following diagnosis was correlated with tumor recurrence and survival of the patient.

\section{Satisfaction with Voice Scale}

This questionnaire was created by Blood and used in two of his research projects concerning psychosocial adjustment of laryngectomees (Blood et al., 1992; Blood et al., 1994). It consists of only three questions (Appendix D). One question asks about speech 
therapy and the other two ask laryngectomees to rate their communication and ability to be understood. The questionnaire was found to correlate highly with psychosocial adjustment as measured by the PAIS and the PAIS-SR, to such an extent that Blood et al. (1992) suggest that perception of voice may predict overall adjustment.

\section{Psychosocial Adjustment to Illness Scale (PAIS) and Psychosocial Adjustment to Illness Scale- Self Report (PAIS-SR)}

Investigators in the area of psychiatric disorders can find a whole array of standardized measures to suit their purposes. For example, there are measures of depression, coping, affect, distress, social support, and others. However, there are not many measures available for investigators of medical disorders in spite of the fact that medical disorders may have psychosocial components (Derogatis \& Derogatis, 1990). For example, although such measures as the Affect of Balance Scale, Ways of Coping Checklist, and Beck Depression Inventory have been used in research with laryngectomees, none of these measures have been normed on cancer patients. The PAIS is a 46-question structured interview which was created by Derogatis and Derogatis (1990) to assess subjects' psychosocial adjustment to a variety of illnesses. Its questions cover seven domains related to psychosocial function. The first is Health Care Orientation, which assesses adjustment to illness and its treatment. The second is Vocational Environment, which asks questions about variables such as vocational satisfaction and interest, and how much work time was lost due to illness. The third is Domestic Environment, which assesses the adaptation of the family toward the illness and the quality of family relationships. The fourth is Sexual Relationships, which estimates the quality of changes that may have taken place in sexual function or relationships. The fifth is Extended Family Relationships, which measures disruption that may have occurred in the extended 
family as a result of the illness. The sixth is Social Environment, which assesses whether the patient's social and leisure activities have decreased as a result of the illness or its treatment. The seventh is Psychological Distress, which measures anxiety, body image problems, depression and other dysphoric feelings associated with the illness.

In creating the PAIS-SR, Derogatis and Derogatis (1990) rephrased the PAIS so that it could be read and answered by the patient. Derogatis (1986) indicated that although the flexibility of the interview format could not be retained, every effort was made to match the PAIS and the PAIS-SR question for question. Norms are available for, among others, lung cancer patients, renal dialysis patients, burn patients, cardiac patients, and mixed cancer patients (Derogatis \& Derogatis, 1990). The PAIS and the PAIS-SR have been utilized in an abundant number of clinical research protocols and clinical trials that have confirmed the tools' predictive validity and reliability (Derogatis \& Fleming, 1996).

Internal consistency reliability coefficients for the PAIS are shown for a sample of 89 lung cancer patients, according to each domain of the PAIS in Table 2.

\section{TABLE 2}

Psychosocial Adjustment to Illness Scale (PAIS) Internal Consistency Reliability Across Subtest Domains

\begin{tabular}{|ll|c|}
\hline \multicolumn{1}{|c|}{ PAIS Domain } & Lung Cancer Coefficients \\
\hline I. & Health Care Orientation & .83 \\
II. & Vocational Environment & .87 \\
III. & Domestic Environment & .68 \\
IV. & Sexual Relationship & .93 \\
V. & Extended Family & $.12^{*}$ \\
VI. & Social Environment & .93 \\
VII. & Psychological Distress & .81 \\
\hline
\end{tabular}

*This subscale was subsequently revised (Derogatis \& Derogatis, 1990). 
A trial of predictive validity showed that the PAIS discriminates between the psychosocial adjustment levels of patients with serious illness and a control group of individuals who are not ill. Domain scores and total scores on the PAIS were compared for 120 patients screened and confirmed for lung cancer, and 86 controls screened and found negative. Differences between the two groups were significant on five of the seven domain scores and on the total scores (Derogatis \& Derogatis, 1990).

A convergent validation process was performed in which 27 breast cancer patients took the PAIS and several other widely used psychological measures. Correlations between PAIS total score and the other measures are given in Table 3.

\section{TABLE 3}

Correlation of PAIS with Other Commonly Used Psychological Measures

\begin{tabular}{|l|l|c|}
\hline External Criterion Measure & Description & $\begin{array}{c}\text { Correlation with } \\
\text { PAIS }\end{array}$ \\
\hline $\begin{array}{l}\text { Global Adjustment to Illness Scale } \\
\text { (GAIS) }\end{array}$ & Global rating scale & .81 \\
\hline SCL-90-R General Severity Index & Measure of distress & .60 \\
\hline Affect Balance Scale (ABS) & Measures mood, affect & .69 \\
\hline $\begin{array}{l}\text { Patient's Attitudes, Information, and } \\
\text { Expectancies Scale (PAIE) }\end{array}$ & $\begin{array}{l}\text { Measures attitudes, expectations } \\
\text { concerning illness }\end{array}$ & .64 \\
\hline
\end{tabular}

Each domain of the PAIS-SR is scored separately and assigned its own "Domain T-score." Domain T-scores are added together to create a Raw Score. The raw score is then converted into a composite T-score which constitutes the overall score on the PAIS-SR. In the norms for mixed cancer patients, T-scores range from 23 to 75 . A score of less than 62 
indicates that the subject is psychosocially "well-adjusted" and a score of 62 or higher indicates that the subject is "poorly adjusted" relative to other cancer patients.

\section{SUMMARY}

In 1996, there were 11,600 new cases of laryngeal cancer in the United States; 9200 of these cases were in males and 2400 in females, giving a male to female proportion of almost 4:1 (American Cancer Society, 1996). Proportions of males to females in the laryngectomy studies looked at by the Principal Investigator are variable, ranging from 1:1 to 5:1. Many studies in the medical literature include only male laryngectomees. Although the ages of peak incidence for laryngeal cancer are from 60 to 79, average ages in the studies cited in this thesis are from 58 to 64 .

There is extensive information in medical literature concerning tobacco and alcohol use in head and neck cancer patients. To summarize this information, $91 \%$ to $98 \%$ of head and neck cancer patients smoked prior to their cancer diagnosis. The most common amount smoked is 1 or more packs of cigarettes per day. Most head and neck cancer patients stop smoking following medical treatment, but between $2 \%$ and $19 \%$ continue to smoke.

Doctors have estimated that from $24 \%$ to $60 \%$ of their laryngeal cancer patients were alcoholic. Alcohol intake of head and neck cancer patients is higher, at 65 to 69 grams per day, than that of the general population, at 29 grams per day. Seven percent of the general population and $28 \%$ of laryngeal cancer patients report 21 or more drinks per week. Using the Michigan Alcoholism Screening Test, $40 \%$ of laryngeal cancer patients were classified as "probably" alcoholic. 
There is also a great deal of information in communication disorders literature about the psychosocial adjustment of laryngectomees. The factors that first caught researchers' attention were the effect on psychosocial adjustment of loss of voice and ability or inability to learn esophageal speech. Some researchers believe that positive feelings about one's alaryngeal voice are a good overall predictor of good psychosocial adjustment.

Other factors that seem to have a positive effect on adjustment are preoperative counseling, internal locus of control, and adequate social support. The factor that seems to have a negative effect on adjustment is respiratory problems that are common for the first year or two following surgery. Finally, the factors that researchers believe have an effect on adjustment, but do not agree whether the effect is positive or negative, are type of medical treatment and time post-onset of surgery.

Successful rehabilitation following laryngectomy is a challenging undertaking under the best of circumstances. Unfortunately, due to the very nature of the etiology of head and neck cancer, many laryngectomees are at risk for less than optimal rehabilitation because of their history of substance dependency. Not only must they grapple with this issue, but also with the disfigurement and dysfunction resulting from cancer surgery. The medical community acknowledges the importance of confronting substance dependency, but its impact on rehabilitation outcomes, specifically psychosocial adjustment, has yet to be defined. This study focused on the question of whether lifestyle change (cessation or reduction of substance use) occurs for most laryngectomees, and whether the effect of lifestyle change has positive or negative implications for psychosocial adjustment.

The major contribution of this study is that it is the first effort at bringing the undiscussed topic of substance abuse, specifically alcohol abuse, into the field of 
communication disorders. Alcohol abuse is a problem that pervades one's life, affecting cognition, health, and behavior. It is important for communication disorders specialists to understand how physical, psychological, and social functioning are impacted by substance abuse in order to contribute to an improved rehabilitation outlook for laryngectomees. 


\section{CHAPTER III}

\section{METHODS}

The main purpose of this study was to examine level of psychosocial adjustment in a limited sample of laryngectomees. The study sought to describe general characteristics of the sample including age, gender, dominant method of speech production, and support group participation, as well as specific characteristics of the sample including self-report of alcohol and tobacco use prior to cancer diagnosis and following laryngectomy (i.e., current status). The relationships between continuing tobacco and alcohol use, reported satisfaction with voice and level of psychosocial adjustment were also examined.

\section{SUBJECTS}

\section{Subject Criteria}

The relatively low incidence of laryngectomy and limitations in potential sample size in the Pacific Northwest were two factors that precluded the collection of a random sample of subjects for this study. Volunteer participation by the laryngectomy community at large was pursued to gather data on 25 subjects, allowing adequate power for completion of selected statistical analyses. The following criteria were used in the selection of participants:

1. Subjects had undergone total laryngectomy. Methods of surgical procedure and attending surgeon were not factors in subject selection. Because the design of the study included administration of a questionnaire concerned with satisfaction with alaryngeal voice, it 
was necessary to eliminate partial laryngectomees, who still use laryngeal voice, as potential subjects.

2. Subjects were required to communicate by using some form of speech, such as esophageal, tracheoesophageal, or artificial larynx. Method of speech was noted, but was not a factor in subject selection as (a) a sufficient subject sample size could not be obtained if the investigator controlled for method of speech utilized, (b) many laryngectomees use more than one method of communication, and (c) the study sought to determine general characteristics of the population including methods of speech utilized.

3. Subjects' gender and age were not factors in subject selection as it was judged that this would limit the investigator's ability to obtain a sufficient sample size. While the study did not control for these factors, information regarding age and gender was obtained for each subject as a part of demographic data collection. Furthermore, data would be calculated for the entire subject group as well as for male and female subgroups.

\section{Subject Recruitment and Subject Consent}

Subjects were recruited through community agencies providing laryngectomy habilitation within the Pacific Northwest. Specifically, subjects were recruited in one of three ways. The first method of subject recruitment involved sending a letter (Appendix A) written by the Principal Investigator, notifying potential subjects of the study, its purpose and procedures, and requesting potential subjects to contact the Principal Investigator if they were interested in participating. The second method of subject recruitment involved a brief advertisement in an American Cancer Society's newsletter that reportedly described the study and requested interested parties to contact the Principal Investigator. It is noted that this advertisement was placed by an American Cancer Society officer without advance permission 
of the Principal Investigator. The third method of subject recruitment involved a verbal presentation about the study by the Principal Investigator at a monthly meeting of the Oregon branch of the New Voice Club held at the American Cancer Society office in Portland. Subjects who (a) met the study's criteria for inclusion, (b) agreed to participate, (c) signed a letter of consent to participate (Appendix B) approved by Portland State University's Human Subjects in Research Committee (Appendix C), and (d) completed the study protocol were included in the study.

\section{Final Subject Distribution}

Data for the first 25 consecutive subjects who met subject criteria, agreed to participate in the study, and completed the study protocol were to be included in the study. Subject recruitment was initiated October 1996. A combined number of 64 potential subjects were identified through the Oregon New Voice Club with an additional unspecified number of potential subjects that read the advertisement in the monthly newsletter of the American Cancer Society. Return response rate for request to participate, regardless of agreement or decline to participate, was $39 \%$. Of the total number of $64+$ potential subjects, no one responded but declined to participate while 25 responded and agreed to participate.

The 25 individuals who agreed to participate were provided the study questionnaires for completion. Three subjects, however, failed to complete and return the study protocol. One was due to untimely demise while the other two potential subjects who had agreed to participate, failed to provide a reason for not returning the questionnaires despite one postcard reminder. Final response rate for those who had agreed to participate was $88 \%$. In December 1996 , following $2^{1 / 2}$ months of attempted subject recruitment, it was felt that the subject recruitment potential of the Pacific Northwest was exhausted for those subjects who met study 
criteria and would agree to participate. Data collection was ceased at that time with data collected on a total of 22 laryngectomees. One potential subject's data, however, could not be used since the subject did not indicate type of alaryngeal speech, which was a requirement for inclusion in the study. The final subject distribution according to age and gender is presented in Table 4.

\section{TABLE 4}

Final Subject Distribution According to Age and Gender $(N=21)$

\begin{tabular}{|l|c|c|c|}
\hline & $n$ & $\begin{array}{c}\text { Average Age } \\
\text { (Years/Months) }\end{array}$ & Age Range in Years \\
\hline Males & 16 & 71.8 & $60-85$ \\
\hline Females & 5 & 74.6 & $72-79$ \\
\hline
\end{tabular}

\section{PROCEDURES}

\section{Data Collection}

The researcher provided a packet containing consent form (Appendix B), instructions (Appendix D), and questionnaires to each subject who agreed to participate. Data were collected from each subject independently. Nineteen of the subjects received the packet in the mail and returned the completed study protocol questionnaires in an included self-addressed stamped envelope to the Principal Investigator. Six of the subjects were handed sets of questionnaires at the New Voice Club meeting previously mentioned after signing the consent to participate. One additional subject, after receiving the questionnaires in the mail and agreeing to participate, reported inability to independently complete the study protocol questionnaires secondary to visual impairment. Data collection for this subject was completed 
in her own home via the Principal Investigator reading study protocol items aloud and documenting subject's response to each item.

It is estimated that the entire study protocol took subjects approximately 45 minutes to 60 minutes to complete. Subjects were encouraged to fill out each questionnaire as completely as possible. Subjects were identified solely by number on each questionnaire form.

\section{Study Measurement Tools}

Subjects selected for this study completed the following: (a) Demographic Questionnaire (Appendix E), (b) Lifestyle Questionnaire (Stevens, 1983) (Appendix F), (c) Satisfaction with Voice Scale (Blood et al., 1994) (Appendix G), and (d) Psychosocial Adjustment to Illness Scale-Self Report (PAIS-SR) (Derogatis \& Derogatis, 1990). The following provides information about each tool regarding content, scoring, and validity.

Demographic Questionnaire. A questionnaire was composed for the purposes of this study to elicit information regarding subjects' gender, age, month and year of laryngectomy surgery, type of speech used, support group membership, and self-report of overall health (Appendix E). The literature reflects that questions such as these are frequently asked during studies about the effects of laryngectomy. Some of these variables are believed to be related to psychological state, perceived quality of life, and level of adjustment (Blood et al., 1992;

Gates et al., 1982a; Mohide et al., 1992; Mathieson et al., 1991; Stam et al., 1991). Questionnaires were coded according to date completed and subject number.

Lifestyle Questionnaire. The Lifestyle Questionnaire, based on a questionnaire by Stevens et al. (1983), was used to measure the variables of "lifestyle" and "lifestyle change." Lifestyle for the purposes of this study is defined as smoking and/or drinking habits of a 
individual, and lifestyle change for the purposes of this study is defined as changes in the smoking and/or drinking habits of an individual. Validity data on the original Lifestyle Questionnaire developed by Stevens et al. were unavailable. It is noted, however, that the format of questions used in the questionnaire approximate guidelines used by the United States Department of Health and Human Services for measuring frequency and amount of alcohol consumption to determine if an individual can be considered a light, moderate, or heavy drinker.

For the purposes of this study, the Lifestyle Questionnaire, printed in relatively large font size, was adapted by dividing it into two parts: Part A consisted of questions about lifestyle prior to cancer diagnosis. Part B consisted of questions about current lifestyle. The questionnaire was set up in a forced-response format that required subjects to circle or check a response. For example, a Part B Question asked:

If you currently smoke, how much do you smoke? up to $1 / 2$ pack per day $1 / 2$ pack to 1 pack per day 1 to 2 packs per day more than 2 packs per day I smoke cigars (How many per day? I smoke a pipe (How much per day?

It was realized that there was a limitation to the study in that information was obtained through self-report. Elicitation of objective information regarding drinking and smoking behaviors was beyond the scope of this project.

Satisfaction with Voice Scale. The Satisfaction with Voice Scale (Appendix G), created by Blood et al. (1992), was used in this study without change to discover subjects' perceptions regarding their speech. This tool consists of three questions, printed in large font size for use in this study. The first question is open-ended and allows subjects to provide 
pertinent comments about how they are communicating. Responses could be categorized as positive, neutral, or negative. Examples of positive responses are: "fine", "just great"; neutral responses are "OK," "What do you think?"; negative responses are "not very good," "Can you understand me? My wife can't."

The second question, also open-ended, inquires about subjects' thoughts regarding the benefits of speech therapy. Response to Question 2 can be categorized as positive, negative, or neutral. For example, "it helped me get some control back" would be considered a positive response while "I just couldn't get it" would be considered a negative response (Blood, 1994, p. 40). On questions 1 and 2, a positive rating scored 1 , a neutral rating scored 2 , and a negative rating scored 3 .

The third question, set-up in a Likert-type 10- point scale, required subjects to circle a number that reflected judgment of how well they are able to make themselves understood with 1 being "understood all the time" and 10 being "rarely understood." Only each end of the continuum was anchored by a verbal label. Subjects' scores from questions 1,2 , and 3 were added together to create a single number called Overall Satisfaction with Voice, ranging from a minimum of 3 to a maximum of 16 . This number was used to perform a correlation coefficient. Satisfaction with Voice Scale forms were coded by subject number.

Psychosocial Adjustment to Illness Scale-Self Report (PAIS-SR). The PAIS-SR, created by Derogatis and Derogatis (1990), was used in this study without change. Copyright restrictions prohibited inclusion of a sample of the test form in the Appendix Section of this document. This tool is a widely used 46-item questionnaire that helps to assess adjustment level to illness. It is normed for populations with various illnesses and conditions such as burns, essential hypertension, and cardiac difficulties. For the purposes of this study, the test 
protocol and norms for "mixed cancer patients" were utilized. The 46 items are divided over seven domains: health care orientation, vocational environment, domestic environment, sexual relationship, extended family, social environment, and psychological distress. Each of the 46 questions is set up so that subjects circle the response that most closely matches their experience. For example, Question 3 from Section III "Domestic Environment" asks: "How much has your illness interfered with your work and duties around the house?" The subject has the opportunity to respond "not at all," "slight problems, easily overcome," "moderate problems, not all of which can be overcome," or "severe difficulties with household duties." Question 7 from Psychological Distress Section asks the subject to choose from among four answers that range from "not at all" to "extremely" when presented with the statement: "Recently, have you been concerned that your illness has caused changes in the way you look that make you less attractive."

The PAIS-SR was administered according to guidelines given for mailed administration and scored according to the test manual protocol. Test results are considered valid if up to nine questions are left unanswered. The manual provides guidelines for calculating a score for each domain. Domain scores are added together to generate a raw score that is used to obtain a T-score. A subject's T-score indicates level of psychosocial adjustment to illness. Potential T-scores range from 23 to 75 for mixed cancer patient norms. Higher scores indicate poor adjustment while lower scores indicate good adjustment. According to the administration manual, T-scores of 62 or higher indicate poor adjustment and psychological distress (Derogatis \& Derogatis, 1990). 


\section{DATA ANALYSIS}

\section{Descriptive Statistics}

Compilation of responses to the four study measures, (Demographic Questionnaire, Lifestyle Questionnaire Part A and Part B, Satisfaction with Voice Scale, and PAIS-SR), enabled completion of the descriptive statistics listed in Table 1, in Chapter I. Additional descriptive analyses allow comparisons of group type (well-adjusted or poorly adjusted) regarding tobacco use, alcohol consumption, and degree of satisfaction with voice.

\section{Inferential Statistics}

The data were gathered to allow for the completion of selected inferential statistics. Specifically, a $t$-test analysis for the difference between two independent means would be performed to determine if a difference would be found between level of adjustment for subjects found to be well-adjusted and level of adjustment for subjects found to be poorlyadjusted as measured by the PAIS-SR. A $t$-test for the difference between two independent means would be used to determine if there was a significant difference between overall levels of adjustment for subjects who report cessation of tobacco or alcohol usage following surgery versus those who report continuation of tobacco or alcohol usage. All t-tests would be followed up by Levene's test for Equality of Variances to determine if these differences were significant at the .05 level. Correlation coefficients would be calculated to determine whether overall satisfaction with voice could predict level of adjustment. The reliability of a measure of this type has not been tested, but in the Blood et al. (1994) study that served as a model for this study, self-perception of voice was found to correlate well with adjustment level on the PAIS-SR and with level of social support. 


\section{CHAPTER IV}

\section{RESULTS AND DISCUSSION}

\section{RESULTS}

Twenty-one laryngectomized subjects participated in this study of the effects of lifestyle change and satisfaction with voice on level of psychosocial adjustment. Subjects were recruited from the Pacific Northwest. Response rate to participate in the study was $39 \%$; response rate for return of completed study protocols was $88 \%$. Signed consent to participate letters and completed study protocols were gathered independently for all subjects. One subject who was unable to see sufficiently for self-administration was administered the study protocol by the Principal Investigator reading aloud study protocol items and scoring the subject's responses. All subjects completed Demographic Questionnaire, Lifestyle Questionnaire, Satisfaction with Voice Scale (Blood et al., 1992), and the PAIS-SR (Derogatis \& Derogatis, 1990). All questionnaires were coded and compiled according to subject number. The Satisfaction with Voice Scale and the PAIS-SR were scored according to their test protocols after data had been collected for all subjects.

In this study, the investigator sought to describe the following: (a) demographic characteristics of a sample of laryngectomees in regard to age, gender, speech production method, and participation in support groups; (b) specific characteristics of the laryngectomized population in regard to alcohol consumption and tobacco use prior to surgery and currently; and (c) reported satisfaction with voice including impression of communication skills, 
perceived benefits of speech therapy, and ability to make oneself understood. Furthermore, the study sought to examine the relationship between the subjects' level of psychosocial adjustment and lifestyle change, specifically cessation of tobacco and/or alcohol consumption, as well as the relationship between level of psychosocial adjustment and degree of satisfaction with voice. The results, grouped according to study measurement tool, are summarized below.

\section{DEMOGRAPHIC QUESTIONNAIRE RESULTS}

The literature suggests that a number of variables can influence psychosocial functioning. Information regarding subjects' gender, age, time post-onset of surgery, and health status was obtained via self-report. Results, shown in Table 5, revealed the following.

\section{Gender and Age Characteristics}

All 21 subjects who participated in the study completed the Demographic Questionnaire. The 21 subjects consisted of 16 males and 5 females. The average age of the subjects was 72.5 years, with a range of ages from 60 to 85 years. Average age of male subjects was 71.8 years, with a range of ages from 60 to 85 years. Average age of female subjects was 74.6 years, with a range of ages from 72 to 79 years.

\section{Time Post-Onset of Laryngectomy}

The average length of time since laryngectomy surgery for all subjects as a group was 167.8 months, or approximately 14 years. For male subjects, the average length of time since surgery was 171.3 months, with a range of 34 to 383 months. For female subjects, the average length of time since surgery was 156.4 months, with a range of 87 to 228 months. 
TABLE 5

Demographic Questionnaire Results for Male Subjects, Female Subjects, and All Subjects

\begin{tabular}{|l|c|c|c|}
\hline Demographic Variable & $\begin{array}{c}\text { Males } \\
n=16\end{array}$ & $\begin{array}{c}\text { Females } \\
n=5\end{array}$ & $\begin{array}{c}\text { All Subjects } \\
N=21\end{array}$ \\
\hline Average age & 71.8 years & 74.6 years & 72.5 years \\
\hline Range of ages & $60-85$ & $72-79$ & $60-79$ \\
\hline Average length of time since surgery & 171.3 months & 156.4 months & 167.8 months \\
\hline Range of time since surgery & $34-383$ months & $87-228$ months & $34-383$ months \\
\hline Type of speech* & & & \\
Esophageal & $8(50 \%)$ & $2(40 \%)$ & $10(48 \%)$ \\
TEP & $4(25 \%)$ & $1(20 \%)$ & $5(24 \%)$ \\
Artificial Larynx & $6(38 \%)$ & $2(40 \%)$ & $8(38 \%)$ \\
\hline Member New Voice Club & $13(81 \%)$ & $5(100 \%)$ & $18(86 \%)$ \\
\hline Health status & & & \\
Excellent & $1(6 \%)$ & $1(20 \%)$ & $2(10 \%)$ \\
Very good & $6(38 \%)$ & $2(40 \%)$ & $8(38 \%)$ \\
Good & $5(31 \%)$ & $2(40 \%)$ & $7(33 \%)$ \\
Fair & $4(25 \%)$ & 0 & $4(19 \%)$ \\
Poor & 0 & 0 & 0 \\
\hline
\end{tabular}

*Some subjects use more than one type of speech.

\section{Method of Speech Production}

As anticipated, some of the subjects indicated that they used more than one method of speech production. The most commonly used speech method for males was esophageal speech (50\%) while females reported equal usage of esophageal speech (40\%) and artificial larynx (40\%). Altogether, 10 subjects use esophageal speech (48\%), 8 subjects use artificial larynx (38\%), and 5 subjects use tracheoesophageal devices for speech production (24\%). 


\section{Support Group Membership}

Of the 21 subjects who participated in the study, 18 subjects $(86 \%)$ reported membership in the New Voice Club, which is a support group for laryngectomees and their families. Percentages calculated according to gender showed that 13 males (81\%) and 5 females $(100 \%)$ were members of the New Voice Club.

\section{Current Health Status}

Subjects were asked in a fixed-response format how they would describe their current health. Possible choices included excellent, very good, good, fair, and poor. Eighty-one percent of subjects responded with excellent, very good or good, thus showing "positive" feelings about their health status, while the remaining $19 \%$, all males, described their health status as fair.

\section{LIFESTYLE QUESTIONNAIRE RESULTS}

The Lifestyle Questionnaire, divided into Part A and Part B, was administered to obtain information about subjects' smoking and drinking behaviors prior to surgery and currently, according to self-report. It was beyond the scope of the study to obtain objective data. Responses to the Lifestyle Questionnaire are summarized in Tables 6 and 7.

\section{Lifestyle Prior To Cancer Diagnosis in Regard to Smoking Behavior}

Twenty of the 21 subjects (95\%) reported that they had smoked prior to their cancer diagnosis, including 15 males (94\%) and 5 females (100\%). The average number of years smoked was 41.25 years for the group as a whole. Male subjects had smoked an average of 40.7 years and female subjects had smoked an average of 42.8 years. Ninety percent of 
subjects (18), or $87 \%$ of male subjects and $100 \%$ of female subjects had smoked one or more packs of cigarettes per day.

\section{Lifestyle Prior To Cancer Diagnosis in Regard to Drinking Behavior}

Twenty subjects (95\%), or 15 males (94\%) and 5 females (100\%), reported alcohol consumption prior to their cancer diagnosis. These were the same subjects who had reported smoking prior to cancer diagnosis. Seventy percent of the entire subject group reported that they had "drunk daily." This group consisted of 9 males and 4 females.

\section{Lifestyle Following Laryngectomy in Regard to Smoking Behavior}

All of the subjects (95\%) except for one male (5\%) reported that they no longer smoked. The one male subject indicated that he consumed on average one-half to one pack of cigarettes per day.

\section{Lifestyle Following Laryngectomy in Regard to Drinking Behavior}

Unlike the results found for smoking behavior, a number of subjects reported continued alcohol consumption. Overall, $38 \%$ of subjects, including 6 males and 2 females, reported cessation or reduction of alcohol consumption since laryngectomy, while another $38 \%$ reported continued daily alcohol consumption averaging two to three units per day.

\section{SATISFACTION WITH VOICE SCALE RESULTS}

The 3-item questionnaire entitled "Satisfaction With Voice Scale" was completed by subjects to gather information about perceived communication skills (Question 1), benefits of speech therapy (Question 2), and ability to make oneself understood (Question 3). Table 8 summarizes subjects' responses to each item. 
TABLE 6

Lifestyle Prior to Cancer Diagnosis

\begin{tabular}{|l|c|c|c|}
\hline & $\begin{array}{c}\text { Males } \\
n=16\end{array}$ & $\begin{array}{c}\text { Females } \\
n=5\end{array}$ & $\begin{array}{c}\text { All Subjects } \\
N=21\end{array}$ \\
\hline Smoked before Dx & $15(94 \%)$ & $5(100 \%)$ & $20(95 \%)$ \\
\hline Drank before Dx & $15(94 \%)$ & $5(100 \%)$ & $20(95 \%)$ \\
\hline
\end{tabular}

\begin{tabular}{|l|l|l|l|}
\hline & \multicolumn{1}{|c|}{$\begin{array}{c}\text { Smoking Males } \\
n=15\end{array}$} & \multicolumn{1}{|c|}{$\begin{array}{c}\text { Smoking Females } \\
n=5\end{array}$} & \multicolumn{1}{|c|}{$\begin{array}{c}\text { All Smoking } \\
\text { Subjects } \\
n=20\end{array}$} \\
\hline Average \# of years smoked & 40.7 & \multicolumn{1}{|c|}{42.8} & \multicolumn{1}{|c|}{41.25} \\
\hline Range in \# of years smoked & $25-54$ & \multicolumn{1}{|c|}{$40-52$} & \multicolumn{1}{|c|}{$25-54$} \\
\hline Amount smoked & & 0 & 0 \\
Up to 1/2 pack/day & 0 & 0 & $2(10 \%)$ \\
$1 / 2$ to 1 pack/day & $2(13 \%)$ & $3(60 \%)$ & $10(50 \%)$ \\
1-2 packs/day & $7(47 \%)$ & $2(40 \%)$ & $8(40 \%)$ \\
>2 packs/day & $6(40 \%)$ & 0 & $2(10 \%)$ \\
Cigars & $2(13 \%)$ & 0 & 0 \\
Pipes & 0 & & \\
\hline
\end{tabular}

\begin{tabular}{|l|l|l|l|}
\hline & \multicolumn{1}{|c|}{$\begin{array}{c}\text { Drinking Males } \\
n=15\end{array}$} & $\begin{array}{c}\text { Drinking Females } \\
n=5\end{array}$ & $\begin{array}{c}\text { All Drinking } \\
\text { Subjects } \\
n=20\end{array}$ \\
\hline $\begin{array}{l}\text { Frequency } \\
1-2 \text { times/year }\end{array}$ & 0 & & \\
$1-2$ times/month & $1(7 \%)$ & 0 & $1(5 \%)$ \\
$1-2$ times/week & $4(27 \%)$ & 0 & $1(5 \%)$ \\
Daily & $10(67 \%)$ & $4(80 \%)$ & $4(27 \%)$ \\
\end{tabular}

\begin{tabular}{|l|l|l|l|}
\hline $\begin{array}{l}\text { Subjects Who Reported } \\
\text { Drinking More than 1 to 2 } \\
\text { Times per Week }\end{array}$ & \multicolumn{1}{|c|}{$\begin{array}{l}\text { Males } \\
n=12\end{array}$} & $\begin{array}{c}\text { Females } \\
n=4\end{array}$ & \multicolumn{1}{|c|}{$\begin{array}{c}\text { All Subjects } \\
n=16\end{array}$} \\
\hline $\begin{array}{l}\text { Amounts in Units Per Day } \\
1 \text { to } 2 \text { units }\end{array}$ & $3(25 \%)$ & 0 & \\
2 to 3 units & $5(43 \%)$ & 0 & $3(19 \%)$ \\
3 to 4 units & $1(8 \%)$ & $1(25 \%)$ & $5(31 \%)$ \\
4 or more units & $3(25 \%)$ & $2(25 \%)$ & $2(13 \%)$ \\
Did not answer & 0 & $2(50 \%)$ & $2(13 \%)$ \\
\hline
\end{tabular}


TABLE 7

Lifestyle Following Laryngectomy Surgery

\begin{tabular}{|l|l|l|l|}
\hline & \multicolumn{1}{|c|}{$\begin{array}{c}\text { Males } \\
n=16\end{array}$} & \multicolumn{1}{|c|}{$\begin{array}{c}\text { Females } \\
n=5\end{array}$} & \multicolumn{1}{|c|}{$\begin{array}{c}\text { All Subjects } \\
N=21\end{array}$} \\
\hline Subjects who smoke & $1(7 \%)$ & 0 & $1(5 \%)$ \\
\hline Amount current smoked & $1 / 2$ to 1 pack/day & & \\
\hline Current drinking frequency & & & \\
Not drinking & $6(38 \%)$ & $2(40 \%)$ & $8(38 \%)$ \\
1 to 2 times/year & 0 & $1(20 \%)$ & $1(5 \%)$ \\
1 to 2 times/month & $1(6 \%)$ & $1(20 \%)$ & $2(10 \%)$ \\
1 to 2 times/week & $1(6 \%)$ & $1(20 \%)$ & $2(10 \%)$ \\
Daily & $8(50 \%)$ & 0 & $8(38 \%)$ \\
\hline
\end{tabular}

\begin{tabular}{|l|l|}
\hline $\begin{array}{l}\text { Subjects Who Reported } \\
\text { Drinking Daily }\end{array}$ & \multicolumn{1}{|c|}{$\begin{array}{r}\text { Males } \\
n=8\end{array}$} \\
\hline $\begin{array}{l}\text { Amounts in Units Per Day } \\
1 \text { to } 2 \text { units }\end{array}$ & $1(13 \%)$ \\
2 to 3 units & $5(63 \%)$ \\
3 to 4 units & 0 \\
4 or more units & $2(25 \%)$ \\
\hline
\end{tabular}

\section{Description of Current Communication Skills.}

Responses to the open-ended question "Describe how you think you are communicating" were tallied as positive, negative, or neutral. Of the 21 subjects, one subject appeared to misunderstand the question as he wrote in the brand name of this electrolarynx.

This subject's response was tallied as neutral. The majority of subjects ( $81 \%$ ) made positive comments regarding their communication skills. This group of "positive thinkers" consisted of 12 males and 5 females. 
TABLE 8

Satisfaction with Voice Scale Results

\begin{tabular}{|c|c|c|c|}
\hline & $\begin{array}{c}\text { Males } \\
n=16\end{array}$ & $\begin{array}{c}\text { Females } \\
n=5\end{array}$ & $\begin{array}{l}\text { All Subjects } \\
\qquad N=21\end{array}$ \\
\hline $\begin{array}{l}\text { Self-perception regarding speech } \\
\text { status (Question 1) } \\
\text { Positive } \\
\text { Negative } \\
\text { Did not Answer }\end{array}$ & $\begin{array}{l}12(75 \%) \\
3(19 \%) \\
1(6 \%)\end{array}$ & $\begin{array}{l}5(100 \% \\
0 \\
0\end{array}$ & $\begin{array}{l}17(81 \%) \\
3(14 \% \\
1(5 \%)\end{array}$ \\
\hline $\begin{array}{l}\text { Perceived benefits of speech therapy } \\
\text { (Question 2) } \\
\text { Positive } \\
\text { Neutral } \\
\text { Negative } \\
\text { Did not have tx } \\
\text { Did not answer }\end{array}$ & $\begin{array}{l}5(31 \%) \\
4(25 \% \\
4(25 \%) \\
3(19 \%) \\
0\end{array}$ & $\begin{array}{l}2(40 \% \\
2(40 \% \\
0 \\
0 \\
1(20 \%)\end{array}$ & $\begin{array}{l}7(33 \%) \\
6(29 \%) \\
4(19 \%) \\
4(19 \%) \\
1(5 \%)\end{array}$ \\
\hline $\begin{array}{l}\text { Ability to be understood } \\
\text { Understood all the time } \\
\text { (Ratings of } 1,2,3 \text { ) } \\
\text { Understood some of the time } \\
\text { (Ratings of } 4,5,6) \\
\text { Rarely understood } \\
\text { (Ratings of } 7,8,9,10 \text { ) }\end{array}$ & $\begin{array}{l}10(63 \%) \\
5(31 \%) \\
1(6 \%)\end{array}$ & $\begin{array}{l}4(80 \%) \\
1(20 \%) \\
0\end{array}$ & $\begin{array}{l}14(67 \%) \\
6(29 \%) \\
1(5 \%)\end{array}$ \\
\hline
\end{tabular}

\section{Perceived Benefits of Speech Therapy}

Question 2 of the Satisfaction With Voice Scale asked: "How did speech therapy help with adjusting or coping with the cancer?" Subjects' responses were tallied as positive, negative, or neutral. Responses to this question were mixed; $33 \%$ of subjects (7) made positive comments, $19 \%$ (4) made negative comments, and $29 \%$ (6) made neutral comments. Of the remaining 4 subjects, 3 had not received speech therapy and 1 subject failed to respond to the question. 
Self-Rating of Ability to Be Understood.

Question 3 of the Satisfaction With Voice Scale requires the subject to "Rate your ability to be understood" on a scale of 1 to 10 with 1 anchored by the verbal description "Understood all the time" and 10 anchored by the verbal description "rarely understood." Sixty seven percent of subjects (14) rated their speech as understood with little difficulty $(1,2$, or 3$)$. Twenty-nine percent (6) rated their speech neutrally $(4,5$, or 6$)$ in that they appeared to judge their speech as "understood some of the time." Only 1 subject, $5 \%$ of the entire group, reported that his alaryngeal speech was "rarely understood."

\section{PSYCHOSOCIAL ADJUSTMENT TO ILLNESS SCALE-SELF REPORT (PAIS-SR) RESULTS}

The PAIS-SR is a tool used to measure an individual's adjustment to illness, using a 46-item questionnaire. As stated previously, the accompanying protocol manual states that up to nine questions on the test can be left unanswered and the questionnaire results can still be considered valid. Of the 21 subjects that participated in this study, 5 subjects, 2 males and 3 females, left more than nine questions unanswered. Types of questions that were unanswered were most often those from the subscales relating to work, marital relationships, and domestic environment whereas sections related to health care orientation, vocational environment, sexual relationship, extended family, social environment, and psychological distress were typically completed. Guidelines were given in the manual as to how to best deal with unanswered responses. Due to the small subject size, all questionnaires, including those with greater than nine items left blank, were scored and results are reported. No subject left more than 16 questions unanswered. 
Fifteen of the subjects (71\%) were classified as "well-adjusted", with a PAIS-SR score of less than 62 , while $6(29 \%)$ were classified as "poorly adjusted", with a score of 62 or higher. The average score for all subjects was 53.38. Average score for males was 55.3, with a range of 40 to 75 . Average score for females was 47 with a range of 34 to 61 . Table 9 summarizes these findings.

\section{TABLE 9}

Compilation of PAIS-SR Scores for Males, Females, and All Subjects by Averages, Range, and Category

\begin{tabular}{|l|c|c|c|}
\hline & $\begin{array}{c}\text { Males } \\
n=16\end{array}$ & $\begin{array}{c}\text { Females } \\
n=5\end{array}$ & $\begin{array}{c}\text { All Subjects } \\
N=21\end{array}$ \\
\hline Average score & 55.3 & 47 & 53.38 \\
\hline Range of scores & $40-75$ & $34-61$ & $34-75$ \\
\hline Well-adjusted & $10(63 \%)$ & $4(100 \%)$ & $15(71 \%)$ \\
\hline Poorly adjusted & $6(38 \%)$ & 0 & $6(29 \%)$ \\
\hline
\end{tabular}

A t-test for the difference between two independent means was performed to determine if there was a significant difference between the mean scores of the well-adjusted group $(X=49.43$; $S D=8.38)$ and the poorly adjusted group $(X=66.00 ; S D=5.09)$. This was done to assure validation of the test results insofar as the PAIS-SR test manual claims that "well-adjusted" and "poorly-adjusted" subjects can be divided by test score into two distinct groups. A mean difference of -16.56 was found. Levene's Test for Equality of Variances was used to determine if that level of difference was significant. Using an alpha value of .05 , the p-value was found to be significant at the .001 level, thus indicating that the differences between the well-adjusted group and the poorly-adjusted group were not at the level of chance. 


\section{LEVEL OF PSYCHOSOCIAL ADJUSTMENT AND REPORTED CESSATION OF TOBACCO USE AND/OR ALCOHOL CONSUMPTION}

In addition to describing selected characteristics of a group of laryngectomees, this study sought to compare subjects by group type (well-adjusted or poorly adjusted) and lifestyle changes in alcohol and/or tobacco consumption following surgery. Table 10 shows the percentage comparison between adjustment level and current lifestyle.

\section{TABLE 10}

Level of Adjustment as Measured by the PAIS-SR and Current Lifestyle in Regard to Tobacco Use and Alcohol Consumption

\begin{tabular}{|l|c|c|}
\hline & Well-Adjusted & Poorly-Adjusted \\
\hline No current tobacco consumption & $16(76 \%)$ & $4(19 \%)$ \\
\hline Current tobacco consumption & 0 & $1(5 \%)$ \\
\hline No current alcohol consumption & $9(43 \%)$ & $4(19 \%)$ \\
\hline Current alcohol consumption & $6(29 \%)$ & $2(10 \%)$ \\
\hline
\end{tabular}

To test the hypothesis that there would be a significant difference between adjustment levels of subjects who continued to smoke following surgery and subjects who stopped smoking, a t-test for the difference between two independent means was to be performed. However, the test could not be performed due to the fact that only one subject continues to smoke. It is worthy of note that this subject fell into the "poorly adjusted" category.

To test the hypothesis that there would be a significant difference between adjustment levels of subjects who continued to drink daily following surgery and subjects who have ceased drinking, or have less than daily consumption, a t-test for independent samples was performed using the mean PAIS-SR score for subjects who were drinking daily $(X=52.38$; 
$S D=8.55)$, and for those who were not, $(X=54.00 ; S D=11.825)$. A mean difference of -1.625 was found. Levene's Test for Equality of Variances was used to determine that, at an alpha value of .05 , there was no significant difference between the two levels of adjustment.

\section{LEVEL OF PSYCHOSOCIAL ADJUSTMENT AND DEGREE OF SATISFACTION WITH VOICE}

To test the hypothesis that overall satisfaction with voice would predict level of psychosocial adjustment, a correlation coefficient was performed between PAIS-SR score and overall satisfaction with voice score. However, this test failed to find any relationship between the two variables at an alpha level of .05 . Visual inspection of the data reveals that the majority of subjects, regardless of being well or poorly adjusted, responded positively when asked to describe their speech. Table 11 shows the relationship between level of adjustment and degree of satisfaction with voice as determined by question 1 on the Satisfaction with Voice Scale.

\section{TABLE 11}

Level of Adjustment as Measured by the PAIS-SR and Degree of Satisfaction with Voice

\begin{tabular}{|l|c|c|}
\hline & Well-Adjusted & Poorly-Adjusted \\
\hline $\begin{array}{l}\text { Positive description of current } \\
\text { speech status }\end{array}$ & $13(87 \%)$ & $4(67 \%)$ \\
\hline $\begin{array}{l}\text { Negative description of current } \\
\text { speech status }\end{array}$ & $2(13 \%)$ & $1(17 \%)$ \\
\hline
\end{tabular}




\section{DISCUSSION}

\section{Demographic Information}

This sample of laryngectomees appeared to be representative of the laryngectomized population at large in several ways. One way in which it was typical is reflected in the percentage breakdown of males to females. In other studies, the percentage of males subjects ranged from $69 \%$ to $82 \%$ while the percentage of female subjects ranged $18 \%$ to $31 \%$ (Blood et al., 1992; Blood, 1993; Blood et al., 1994; Jay et al., 1991) for subject group data. In the present study, the subject group was comprised of 16 males (76\%) and 5 females (24\%), giving a proportion of about 3:1, males to females.

Another way in which this sample seems to be representative of the population is the percentages of type of speech used. In other studies, use of esophageal voice ranges from $34 \%$ to $54 \%$; tracheoesophageal device from $25 \%$ to $33 \%$; artificial larynx from $15 \%$ to $37 \%$ (Blood et al., 1992; Blood, 1993; Blood et al., 1994). In this study, $48 \%$ of subjects used esophageal voice, $24 \%$ used tracheoesophageal device, and $38 \%$ used artificial larynx.

While there were the above noted similarities in this study's subject group compared to those discussed in the literature, analyses of results revealed several ways in which they differed. Specifically, differences were found in regard to age, time post-onset, and possibly, two other parameters that were not specifically targeted : marital status and employment status. In nine studies of laryngectomees looked at by this researcher, the average age ranges from 58 to 64 years. There is also one study from England in which the average subject age was 68 years. In this study, however, the average age of subjects was 72.5 years. It is unclear why the subjects in this study were so much older on average than subjects in other studies. It 
is possible that younger laryngectomees are still working, and did not feel they had time to volunteer for this study.

Many of the subjects in this study were retirees, who would generally have more time to volunteer. Another possibility is that fewer laryngectomy surgeries are being performed nowadays, resulting in an increased number of aging laryngectomees. The Principle Investigator has information from anecdotal sources that laryngeal cancer is currently being treated more often through radiation and chemotherapy than through surgery.

In any case, it is fairly certain the age difference does not relate to source of subjects, since the subjects in this study were found from the same support groups as those in the studies discussed above. Not all of the researchers involved in those studies reveal the source of their subjects, but some do. Subjects were recruited from the International Association of Laryngectomees (IAL) and Lost Chord Clubs in the United States (Blood, 1993; Blood et al., 1994), from an oncology institute, from hospitals, tumor registries, and local government records (Gates et al. 1982a; Jay et al., 1991; Pradier et al., 1993 Stevens et al., 1983; Freudenheim et al., 1992). Subjects recruited from the IAL and Lost Chord Clubs had an average age of only 61 and 64 years, respectively. Younger samples such as these have a higher likelihood of being married, and of being employed. The age factor is reflected in the average length of time post-onset for subjects of other studies compared with this one. For the Jay et al. (1991) study, average length of time post-onset was 93.6 months, or 7.8 years; for the Blood et al. (1992) study, the average was 49 months, or 4.1 years; for the Blood (1993) study, it was 43 months, or 3.6 years; for the Blood et al. (1994) study, it was 38 months, or 3.2 years. For this study the average length of time post-onset was 167.8 months, or 14 years! It is possible that for some subjects, the cancer experience has become secondary to other 
stressful life experiences such as widowhood, caregiving to a sick family member, loss of family or friends to death, or another type of chronic or acute illness. However, coping and adjustment to such stressful life experiences that are common for people over 65 are not measured in the PAIS-SR, that focuses on the effects of a long-past cancer experience.

\section{Tobacco Use and Alcohol Consumption}

Based on the literature, it was predicted that $75 \%$ or more of the subjects would report that they had smoked cigarettes at the rate of at least 1 pack per day prior to cancer diagnosis. In fact, $95 \%$ of subjects smoked, and $90 \%$ of these smoked at least one pack per day. Following laryngectomy surgery, however, only one subject (5\%) continues to smoke. The results of this study are consistent with previous research indicating that $2 \%$ to $19 \%$ of laryngectomees smoke.

General findings of this study in relation to tobacco use and alcohol consumption following surgery were that larger numbers of subjects stopped smoking than stopped drinking. Anecdotal information may illuminate the reason for this. During the subject search process for this study, subjects contacted the Principal Investigator by letter or phone to indicate willingness to participate. Upon being warned that they would be asked to volunteer personal information, including smoking and drinking habits, several subjects wrote or commented that they had been told by their doctor to stop smoking, but no similar injunction was made for drinking alcohol. If other subjects were also given the same advice, it would not be surprising that many more subjects continue to drink than continue to smoke. In fact, although smoking is by far the overwhelming risk factor for laryngeal cancer, alcohol too plays a prominent role, and a combination of the two has a synergistic effect on risk (Stevens et al., 1983; Maier et al., 1992; Freudenheim et al., 1992). 
It was predicted that at least $75 \%$ of subjects would report that they had drunk alcohol and that they had consumed four or more units daily prior to cancer diagnosis. In fact, $95 \%$ of subjects reported that they drank alcohol, and of these, $70 \%$ consumed alcohol daily while only $25 \%$ consumed four or more units per day. According to United States Department of Health and Human Services (USDH\&HS) guidelines, moderate drinking is defined as one drink per day for women and two for men. Their definition of a drink is similar to that of a unit in this study, a drink consisting of 12 ounces of beer, 5 ounces of wine, or 1.5 ounces of hard liquor. By the standards of the USDH\&HS, $69 \%$ of the subjects in this study who drank daily pre-diagnosis would be considered heavy drinkers, having drunk more than two units per day. Even now, $38 \%$ of subjects consume alcohol daily, but since they are older, they fall under the USDH\&HS guideline for elderly drinkers, in which moderate drinking is considered to be one drink per day. By these standards, $88 \%$ of subjects who drink daily would be considered heavy drinkers. They represent $33 \%$ of the total number of subjects. This may seem like a small percentage, but the rate of alcoholism in the older population is generally estimated to be about $2 \%$ to $10 \%$ (Traxler, 1991; Colsher \& Wallace, 1990; Egbert, 1993). It appears that even many years post-onset, a large percentage of laryngeal cancer survivors continue to drink heavily compared to the general population of community-based older people. They drink heavily in spite of the existence of factors such as health problems and lifestyle change, that have been shown to be motivations to cessation of alcohol use (Hermos, LoCastro, Glynn, Bouchard, \& De Labry, 1988). Because of their heavy drinking, these cancer survivors are at risk for higher rates of cognitive dysfunction, learning and memory deficits, depression, major illness, falls and accidents, and decreased life satisfaction, social support, and functioning for activities of daily living (ADL) (Colsher \& Wallace, 1990; Curtis, 
Geller, Stokes, Levine, \& Moore, 1989; Egbert, 1993; Graham, 1986). Even those persons who are drinking less are at risk because of the less efficient metabolism of alcohol with age and possible interactions with prescription and non- prescription drugs (Egbert, 1993; Welte \& Mirand, 1995).

\section{Psychosocial Adjustment Level and Use of the PAIS-SR Findings}

A larger percentage of the subjects in this study than other similar studies may be widowed or unmarried. In the Blood et al. (1992) study, 54\% of subjects were married and $29 \%$ widowed; in the Blood (1993) study, $63 \%$ were married and $21 \%$ widowed; and in the Blood et al. (1994) study, $83 \%$ were married and $17 \%$ widowed or divorced. Although marital status was not among the data collected in this study, many of the subjects returned letters with their questionnaires or wrote comments on the PAIS-SR about their status as a widow or widower, particularly in Domain Subscale 4 which focuses on sexual relationship. For example, subject \#2, who was interviewed in person, indicated that she was widowed. She did not fill in Subscale 4. Subject \#3 wrote the comment "section IV- not that kind of 'partner," and did not answer the questions. Subject \#4, who did not answer the questions in subscale 4, wrote above that section "I was a widower." Subject \#12 wrote "Died two years ago" next to a question asking about her relationship with her spouse. She did answer the questions in subscale 4, but since the PAIS-SR specifically targets the period of the previous month, her answers to these questions are invalid. Subject \#15, in lieu of answering the questions in subscale 4, wrote "NA" next to each one, but did not indicate the reason for doing so. Subject \#19 did not answer the questions in subscale 4, and wrote "My husband passed away 2 month [sic]( after my operation I'm still single." Subject \#23 did not answer the questions in subscale 4, but filled in the demographic information on the instruction page of the PAIS-SR. He 
indicated that his marital status was "widower." Subject \#24 wrote "I was a widow I still am" next to a question about her relationship with her spouse. She did not answer the questions in subscale 4. In addition, subjects \#1 and \#5 did not answer the questions in subscale 4, but wrote no comments. This brings the total of subjects who are widowed and/or did not answer the subscale 4 questions to 10 , or $48 \%$ of subjects. There may be other subjects who answered the questions, thinking of some time in the past when their spouse was still living, but their answers would be considered invalid according to the scoring and validity protocol detailed in the PAIS-SR manual.

As for retirement, in the same studies cited above, the percentage of retired subjects was $29 \%, 29 \%$, and $30 \%$. However, in this study, a majority of subjects were retired, and many did not answer questions in subscale II, that relates to vocational environment, or they answered the questions in reference to some time in the past. Subjects $\# 1, \# 5$, \#19, and \#23 did not answer some or all of the questions but wrote no comments. Subject \#3 wrote "section II does not concern me;" subjects $\# 7$, \#12, \#18, and \#20 wrote "retired" on the instruction page of the PAIS-SR. Subject \#10 wrote "Note: I am retired, have been for 11 years. I'm answering for the period about 1978. Ignore it if that time has no meaning." Subject \#14 wrote "I was retired before my operation so my job don’t [sic] enter in to it." Subject \#22 wrote "Been retired for past 10 years unable to answer this section" and subject \#24 wrote "I had to quit work I was 60 years old." This makes a total of 13 subjects, or $62 \%$, who indicated they were retired and/or did not answer questions in subscale 2.

The other section of the PAIS-SR where many questions remained unanswered was Subscale 3, Domestic Environment. Six subjects did not answer from one to four of the eight questions in that subsection. It could be gathered from comments that 5 of the subjects live 
alone. In fact, it must be expected that some of the subjects live alone, since $30 \%$ of people over 65 live alone (Egbert, 1993). For example, subject \#2, who was interviewed in person, indicated that she lived alone. Subject \#19 wrote "I moved away from my family to take care of a sister who has since died. I have not moved back" and subject \#13 simply wrote "N/A" by the question in subscale 3 that asks about the subject's relationship with people living together in the same house. Here again, at least one subject answered questions about a time in the past, and not the past 30 days as instructed on the PAIS-SR. Subject \#19 answered questions about her relationship with her spouse and others living in the same house, but indicated that she lived alone.

A tool such as the PAIS-SR may not be appropriate for a sample such as this, where most of the subjects are retired, where nearly half the subjects are widowed, and nearly a quarter of the subjects live alone. Too many domains of the PAIS-SR simply cannot apply to such subjects, who, when the questionnaire is administered, either do not answer the questions, or answer about a time in the past more than 30 days ago, rendering their answers invalid. Only 3 subjects answered all 64 questions, and the average number of questions left unanswered by the other 18 subjects was 7.5 . Nine is the maximum number of questions that can be left unanswered and still have a valid result. In addition, the PAIS-SR was normed on a sample with very different characteristics from the one in this study. The average age of the cancer patients on whom the PAIS-SR was normed was $53.1 ; 90 \%$ of them were married and none widowed (Derogatis \& Derogatis, 1990). It is doubtful whether the results of the portion of this study that relates to the PAIS-SR are valid at all.

That said, it is interesting to note that the PAIS-SR results were similar for this study and for three other studies in which the PAIS-SR was used. In the Blood et al. (1992) study, 
$73 \%$ of subjects were well-adjusted and $27 \%$ poorly adjusted, with a group mean score of 50.5. In the Blood (1993) study, $77 \%$ of subjects were well-adjusted and $23 \%$ poorly adjusted, but no group mean was reported. In the Blood et al. (1994) study, $70 \%$ of subjects were welladjusted and $30 \%$ poorly adjusted, with a group mean of 46.7 . In this study, $71 \%$ of subjects were well-adjusted and $29 \%$ poorly adjusted, with a group mean of 53.4 . If we decided to accept the use of the PAIS-SR as valid, perhaps we could conclude that adjustment level does not tend to change with increasing age, retirement, or widowhood.

\section{Satisfaction With Voice}

The Satisfaction with Voice scale yielded results that did not agree well with other studies. In both of the studies where Satisfaction with Voice was used (Blood et al., 1992; Blood et al., 1994) positive answers were found to correlate well with psychosocial adjustment, and self-ratings of well-adjusted subjects were found to be more positive than those of poorly-adjusted subjects. In this study, there was no relationship found between positive overall satisfaction with voice and psychosocial adjustment; in addition, average self-ratings of well- and poorly-adjusted subjects were not different from each other. One possible reason for this is the small size of this study. Another might be that, in the long period of time post-onset that was typical for the subjects in this study, friends and family had grown accustomed to subjects' mode of communication, resulting in fewer communication problems for both well- and poorly-adjusted subjects.

\section{STUDY LIMITATIONS}

There was a potential bias in subject recruitment for the variable of "membership in a laryngectomy support group" in that community members willing to assist in subject 
recruitment were associated with the laryngectomee support group, New Voice Club of Oregon. Another difficulty was in eliciting a sample size that would have given sufficient statistical power. Time limitations for completion of thesis requirements prevented subject recruitment from being as extensive as the Principal Investigator would have liked.

Other limitations exist that bear mention. There are several problems which make the results of the PAIS-SR suspect. Firstly, the age and length of time post-onset of most subjects in this study were different from those of previous studies and from the norming population of the PAIS-SR. Secondly, three variables that were not measured, marital status, employment status, and whether the subjects lived alone or with others, had a high likelihood of being different from those of previous studies and from the norming population of the PAIS-SR. Thirdly, internal inconsistencies in the answers given on the PAIS-SR and the high number of questions left unanswered by many subjects led this researcher to conclude that the PAIS-SR was not an appropriate tool to use for this particular sample of laryngectomees. Any statistical calculations involving the PAIS-SR are therefore inconclusive. The combined findings suggest that despite study limitations, the present research found this sample of older laryngectomees reportedly in good health, satisfied with their current communication status, and living long lives in spite of prolonged alcohol use and past history of cancer and laryngectomy surgery. 


\section{CHAPTER V}

\section{SUMMARY AND IMPLICATIONS}

In the early years of laryngectomee rehabilitation, it was believed that attainment of esophageal voice was the essence of successful rehabilitation. Communication disorder specialists later realized that rehabilitation was a more complex, all-encompassing goal that, of necessity, had to reach into other disciplines in order to treat the whole patient. The person who has experienced head and neck cancer has a very high likelihood of being dependent upon tobacco and alcohol prior to diagnosis. Because of physiological changes brought about during laryngectomy surgery, tobacco is largely forsaken by this population, but alcohol use often continues to be heavy. Characteristics of the alcoholic personality include poor adaptability and coping, unwillingness to cooperate with treatment, and poor ability to relate to others, as well as possible learning and memory deficits resulting from heavy alcohol use. Such characteristics do not lend themselves to successful rehabilitation, and in addition place the laryngectomee at risk for further major illness, depression, poor functioning, and poor mental status among other problems.

This research examined the psychosocial adjustment of a limited sample of laryngectomees. The study focused on the levels of continued tobacco and alcohol use as well as reported satisfaction with alaryngeal voice as potential factors affecting psychosocial adjustment level.

It was found that there were high percentages of subjects who reported having smoked 1 pack per day or more, and drank daily prior to diagnosis. Amounts of alcohol consumed 
were not as large as expected, but were still in excess of what the USDH\&HS considers moderate drinking.

Well- and poorly-adjusted subjects were found to have significantly different scores on the PAIS-SR. Neither satisfaction with alaryngeal voice nor cessation of smoking and drinking behavior were found to be highly related to level of psychosocial adjustment. Given that laryngectomees who continue to drink heavily are at risk for higher rates of neurologic disorders, cardiovascular disease, cancer, liver disease, chronic obstructive pulmonary disease, peptic ulcer, pancreatitis, and affective disorders than persons who do not have alcoholism, and that persons who drink heavily place a disproportional burden on our health care system (Colsher \& Wallace, 1990), further research is needed to clarify the role of heavy alcohol use in the rehabilitation of laryngectomees. There are a number of variables which have been studied for persons with alcoholism, and these same variables could be measured and applied to the head and neck cancer population. These include life satisfaction, functioning for ADLs and Instrumental Activities of Daily Living (IADL), social support, depression, major illness, mental status, stress, self-care and nutrition, falls and accidents, and complaints such as sleep disorders (Colsher \& Wallace, 1990; Graham, 1986; Curtis et al. 1989; Welte \& Mirand, 1995). In addition, the CAGE screening questionnaire and the MAST have been found effective with the older population (Egbert, 1993; Curtis et al., 1989; Willenbring et al., 1987). (CAGE is an acronym for the four questions on the questionnaire; it has no other name.)

The scope of this type of research should be enlarged to include the entire head and neck cancer population; in this way the sample could be enlarged. Males, females, and various age groups should be carefully separated, as all of these show different trends in drinking 
behavior. Control groups should also be used to measure differences between the head and neck cancer population and the general population.

As members of the professional health care team, we should increase our awareness of how heavy alcohol use affects our patients so that we can have a better understanding of how to help these patients rehabilitate successfully. 


\section{REFERENCES}

Ackerstaff, A. H., Hilgers, F. J. M., Aaronson, N. K., \& Balm, A. J. M. (1994). Communication, functional disorders, and lifestyle changes after total laryngectomy. Clinical Otolaryngology, 19, 295-300.

Ackerstaff, A. H., Hilgers, F. J. M., Aaronson, N. K., Balm, A. J. M., \& Van Zandwijk, N. (1993). Improvements in respiratory and psychosocial functioning following total laryngectomy by the use of a heat and moisture exchanger. Annals of Otology, Rhinology, and Laryngology, 102, 878-883.

American Cancer Society. (1996). Cancer facts and figures -1996. Atlanta, GA: Author.

American Psychiatric Association. (1994). Diagnostic and Statistical Manual of Mental Disorders (4th ed. ). Washington, DC: Author.

Barton, R. T. (1965). Life after laryngectomy. Laryngoscope, 75, 1408-1414.

Blood, G. W. (1993). Development and assessment of a scale addressing communication needs of patients with laryngectomies. American Journal of Speech-Language Pathology, 2, 82-90.

Blood, G. W., Dineen, M., Kauffman, S. M., Raimondi, S. C., \& Simpson, K. C. (1993). Perceived control, adjustment, and communication problems in laryngeal cancer survivors. Perceptual and Motor Skills, 77, 764-766.

Blood, G. W., Luther, A. R., \& Stemple, J. C. (1992). Coping and adjustment in alaryngeal speakers. American Journal of Speech-Language Pathology, 1, 63-69.

Blood, G. W., Simpson, K. C., Raimondi, S. C., Dineen, M., Kauffman, S. M., \& Stagaard, K. A. (1994). Social support in laryngeal cancer survivors: Voice and adjustment issues. American Journal of Speech-Language Pathology, 3, 37-44.

Breeden, J. H. (1984). Alcohol, alcoholism, and cancer. Medical Clinics of North America, 68(1), 163-177.

Breitbart, W., \& Holland, J. (1988). Psychosocial aspects of head and neck cancer. Seminars in Oncology, 15(1), 61-69. 
Byrne, A., Walsh, M., Farrelly, M., \& O'Driscoll, K. (1993). Depression following laryngectomy: A pilot study. British Journal of Psychiatry, 163, 173-176.

Colsher, P. L., \& Wallace, R. B. (1990). Elderly men with histories of heavy drinking: Correlates and consequences. Journal of Studies on Alcohol, 51(6), 528-535.

Curtis, J. R., Geller, G., Stokes, E. J., Levine, D. M., \& Moore, R. D. (1989). Characteristics, diagnosis, and treatment of alcoholism in elderly patients. Journal of the American Geriatric Society, 37, 310-316.

Darvill, G. (1983). Rehabilitation- not just voice. In Y. Edels (Ed. ), Laryngectomy: Diagnosis to rehabilitation (pp. 192-217). Rockville, MD: Aspen Systems Corporation.

David, D. J., \& Barritt, J. A. (1982). Psychosocial implications of surgery for head and neck cancer. Clinics in Plastic Surgery, 9(3), 327-336.

de Maddalena, H., \& Pfrang, H. (1993). Subjective attitudes of laryngectomized patients of the cause of the tumor disease [On-line]. HNO, 41(4), 198-205. Abstract from: MedLine, Item: 93293535.

Derogatis, L. R. (1986). The Psychosocial Adjustment to Illness Scale (PAIS). Journal of Psychosomatic Research, 30 (1), 77-91.

Derogatis, L. R., \& Derogatis, M. F. (1990). The Psychosocial Adjustment to Illness Scale (PAIS \& PAIS-SR): Administration, scoring, \& procedures manual- II. Towson, MD: Clinical Psychometric Research, Inc.

Derogatis, L. R., \& Fleming, M. P. (1996). Psychological Adjustment to Illness Scale: PAIS and PAIS-SR. In B. Spilker (Ed. ), Quality of life and pharmacademics in clinical trials ( $2^{\text {nd }}$ ed.) (pp. 287- 296). Philadelphia: Lippincott-Raven Publishers.

DeSanto, L. W., Olsen, K. D., Perry, W. C., Rohe, D. E., \& Keith, R. L. (1995). Quality of life after surgical treatment of cancer of the larynx. Annals of Otology, Rhinology, and Laryngology, 104, 763-769.

Egbert, A. M. (1993). The older alcoholic: Recognizing the subtle cues. Geriatrics, 48(7), 63-69.

Fried, M. P. (1988a). Carcinoma of the glottis and subglottis. In M. P. Fried (Ed. ), The larynx: A multidisciplinary approach (pp. 471-490). Boston: Little, Brown, and Company.

Gates, G. A., Ryan, W., Cooper, J. C., Lawlis, G. F., Cantu, E., Hayashi, T., Lauder, E., Welch, R. W., \& Hearne, E. (1982a). Current status of laryngectomee rehabilitation: I. Results of therapy. American Journal of Otolaryngology, 3, 1-7. 
Gates, G. A., Ryan, W., Cantu, E., \& Hearne, E. (1982b). Current status of laryngectomee rehabilitation: II. Causes of failure. American Journal of Otolaryngology, 3, 8-14.

Gilmore, S. I. (1986). The psychosocial concomitants of laryngectomy. In R. L. Keith \& F. L. Darley, (Eds. ), Laryngectomy rehabilitation (2nd ed. ) (pp. 425-495). San Diego, CA: College Hill Press.

Graham, K. (1986). Identifying and measuring alcohol abuse among the elderly: Serious problems with existing instrumentation. Journal of Studies on Alcohol, 47(4), 322326.

Hedberg, K., Vaughan, T. L., White, E., Davis, S., \& Thomas, D. B. (1994). Alcoholism and cancer of the larynx: A case control study in Western Washington (United States). Cancer Causes and Control, 5, 3-8.

Hermos, J. A., LoCastro, J. S., Glynn, R. J., Bouchard, G. R., \& DeLabry, L. O. (1988). Predictors of reduction and cessation of drinking in community-dwelling men: Results from the normative aging study. Journal of Studies on Alcohol, 49(4), 363-368.

Hilgers, F. J. M., Ackerstaff, A. H., Aaronson, N. K., Schouwenburg, P. F., \& Van Zandwijk, N. (1990). Physical and psychosocial consequences of total laryngectomy. Clinical Otolaryngology, 15, 421-425.

Himbury, S., \& West, R. (1985). Smoking habits after laryngectomy. British Medical Journal, 291, 514-515.

Johnson, J. T., Casper, J., \& Lesswing, N. J. (1979). Toward the total rehabilitation of the alaryngeal patient. Laryngoscope, 89, 1813-1819.

Karmody, C. S. (1988). The history of laryngology. In M. P. Fried (Ed.), The larynx: A multidisciplinary approach (pp. 3-13). Boston: Little, Brown, and Company.

King, P. S., Fowlks, E. W., Peirson, G. A. (1968). Rehabilitation and adaptation of laryngectomy patients. American Journal of Physical Medicine, 47(4), 192-203.

Kommers M. S., \& Sullivan, M. D. (1979). Wives' evaluation of problems related to laryngectomy. Journal of Communication Disorders, 12, 411-430.

Logemann, J. A. (1983). Evaluation and treatment of swallowing disorders. Austin, TX: Pro-Ed, Inc.

Lowry, W. S. (1975). Alcoholism in cancer of the head and neck. Laryngoscope, 85, 1275-1280. 
Luce, D., Guenel, P., Brugere, J., Leclerc, A., Point, D., \& Rodriguez, J. (1988). Alcohol and tobacco consumption in cancer of the mouth, pharynx, and larynx: A study of 316 female patients. Laryngoscope, 98, 313-316.

Maas, A. (1991). A model for quality of life after laryngectomy. Social Science Medicine, 33, 1373-1377.

Maier, H., Dietz, A., Gewelke, U., Heller, W. D., \& Weidauer, H. (1992). Tobacco and alcohol and the risk of head and neck cancer. Clinical Investigator, 70, 320-327.

Maisel, R. H., \& Cohen, J. I. (1988). Carcinoma of the supraglottis. In M. P. Fried (Ed. ), The larynx: A multidisciplinary approach (pp. 453-470). Boston: Little, Brown, and Company.

Mathieson, C. M., Stam, H. J., \& Scott, J. P. (1990). Psychosocial adjustment after laryngectomy: A review of the literature. Journal of Otolaryngology, 19(5), 331-336.

Mathieson, C. M., Stam, H. J., \& Scott, J. P. (1991). The impact of a laryngectomy on the spouse: Who is better off? Psychology and Health, 5, 153-163.

Morton, R. P., Davies, D. M., Baker, J., Baker, G. A., \& Stell, P. M. (1984). Quality of life in treated head and neck cancer patients: A preliminary report. Clinical Otolaryngology, 9, 181-185.

Nahum, A. M., \& Golden, J. S. (1963). Psychological problems of laryngectomy. Journal of the American Medical Association, 186(13), 1136-1138.

Norris, C. M., \& Cady, B. (1991). Head, neck, and thyroid cancer. In A. I. Holleb, D. J. Fink, \& G. P. Murphy (Eds. ), American Cancer Society Textbook of Clinical Oncology (pp. 306-316). Atlanta, GA: American Cancer Society, Inc.

Perry, A. (1983a). Assessment: What, why, how, and when to measure social, physical, communication, and psychological improvement. In Y. Edels (Ed. ), Laryngectomy: Diagnosis to rehabilitation (pp. 75-106). Rockville, MD: Aspen Systems Corporation.

Perry, A. (1983b). Difficulties in the acquisition of alaryngeal speech. In Y. Edels (Ed. ), Laryngectomy: Diagnosis to rehabilitation (pp. 177-191). Rockville, MD: Aspen Systems Corporation.

Pradier, R., Gonzalez, A., Matos, E., Loria, D., Adan, R., Saco, P., \& Califano, L. (1993). Prognostic factors in laryngeal carcinoma: Experience in 296 male patients. Cancer, $71(8), 2472-2476$. 
Pruyn, J. F. A., de Jong, P. C., Bosman, L. J., van Poppel, J. W. M. J., van den Borne, H. W., Ryckman, R. M., \& de Meij, K. (1986). Psychosocial aspects of head and neck cancer- a review of the literature. Clinical Otolaryngology, 11, 469-474.

Renner, M. J. (1995). Counseling laryngectomees and families. Seminars in Speech and Language, 16(3), 215-219.

Rice, D. H., \& Spiro, R. H. (1989). Current concepts in head and neck cancer. American Cancer Society.

Salmon, S. J. (1986). Adjusting to laryngectomy. Seminars in Speech and Language, 7(1), 67-93.

Shapiro, P. A., \& Kornfeld, D. S. (1987). Psychiatric aspects of head and neck cancer surgery. Psychiatric Clinics of North America, 10(1), 87-100.

Spiegel, J. R., \& Sataloff, R. T. (1993). Surgery for carcinoma of the larynx. In W. J. Gould, R. T. Sataloff, \& J. R. Spiegel (Eds. ), Voice Surgery (pp. 307-337). St. Louis, MO: Mosby-Year Book, Inc.

Stam, H. J., Koopmans, J. P., \& Mathieson, C. M. (1991). The psychosocial impact of a laryngectomy: A comprehensive assessment. Journal of Psychosocial Oncology, 9 (3), 37-58.

Stevens, M. H., Gardner, J. W., Parkin, J. L., \& Johnson, L. P. (1983). Head and neck cancer survival and life-style change. Archives of Otolaryngology, 109, 746-749.

Traxler, A. (1991, Summer/Fall). Older persons and the abuse and misuse of alcohol and drugs. Prevention Forum, pp. 1-7.

U.S. Department of Health and Human Services. (1992, April). Alcohol Alert. Bethesda, MD: National Institute on Alcohol Abuse and Alcoholism, 16(315).

Wallen, V., \& Webb, V. P. (1975). A survey of the background characteristics of 2000 laryngectomees: A preliminary report. Military Medicine, August 1975, 532-534.

Weber, M., \& Reimer, M. (1993). Laryngectomy: Grieving disfigurement and dysfunction. Canadian Nurse, 89, 31-34.

Willenbring, M. L., Christensen, K. J., Spring, W. D., \& Rasmussen, R. (1987). Alcoholism screening in the elderly. Journal of the American Geriatric Society, 35, 864-869. 
APPENDIX A

SUBJECT RECRUITMENT LETTER 
Dear New Voice Club Member:

Would you be willing to donate 30 to 45 minutes of your time to further research about the well-being of people who have had a laryngectomy?

If you have had a total laryngectomy and you use some type of alaryngeal speech as your primary communication method, you are invited to participate in an anonymous survey being done by Susan Nicks, a Master's Candidate in Speech and Hearing Science at Portland State University. She is currently writing a thesis about the "Psychosocial Adjustment of Laryngectomees."

If you want to participate, you will be asked to sign a consent form and fill out three questionnaires, plus give some information about yourself such as age, when you had laryngectomy surgery, and your general health at this time. There are some personal questions on the questionnaires, for example, your smoking and drinking habits, your opinion of your ability to communicate, relationship with your immediate and extended family, relationship with your spouse if you have one, and your feelings about having had cancer. However, since the survey is anonymous, no one will be able to trace who completed which set of questionnaires.

It is hoped that this survey will contribute to an improved rehabilitation outlook for persons who have a laryngectomy. If you are interested in participating, please call, write, or e-mail Susan Nicks to make arrangements. The researcher will meet you at a mutually agreed-upon location, or send you the questionnaires in the mail.

Thank you for your time.

\author{
Susan Nicks \\ 4107 S.E. Washington Street \\ Portland, OR 97214 \\ (503) 236-1651 \\ e-mail: atsuonicki@cenornet.com
}

I am planning to attend the New Voice Club meeting October $19^{\text {th }}$. If it is convenient for you, you may fill out my questionnaires at that time, or make an appointment to fill them out. Thank you!

NOTE: I received your name and address from Dick Guarnero, President of Oregon New Voice Club. I have no plans to use the information he gave me for any purpose other than that stated above.

If you have concerns or questions about this study, lease contact the Chair of the Human Subjects Research Review Committee and Sponsored Projects, 105 Neuberger Hall, Portland State University, (503) 725-3417. 


\section{APPENDIX B}

WRITTEN CONSENT FORM 


\section{Written Consent Form}

I , agree to take part in this research project on laryngectomy.

I understand that the study involves answering some questions about my feelings, family life, relationship with my spouse, and smoking and drinking habits which I may consider personal and private.

I understand that I may experience an inconvenience of time commitment (about 30 minutes) because of this study.

I will not be paid for my participation.

I may not receive any direct benefit from taking part in this study. However, I understand that the intention of the study is to increase knowledge that may help others in the future.

Susan Nicks, the principal researcher, has offered to answer any questions I have about the study, and what I am expected to do.

She has promised that all the information I give will be kept confidential, and that the names of all people participating in the study will be kept anonymous.

I understand that I do not have to take part in this study and that refusal or agreement to participate will not affect my relationship with Portland State University.

I have read and understand the above information and agree to take part in this study.

Signature

Date

If you have concerns or questions about this study, lease contact the Chair of the Human Subjects Research Review Committee and Sponsored Projects, 105 Neuberger Hall, Portland State University, (503) 725-3417. 


\section{APPENDIX C}

PORTLAND STATE UNIVERSITY'S USE OF HUMAN SUBJECTS IN RESEARCH APPROVAL LETTER 


\section{OFFICE OF GRADUATE STUDIES AND RESEARCH \\ Research and Sponsored Projects}

DATE: $\quad$ August 16, 1996

TO: $\quad$ Susan Nicks

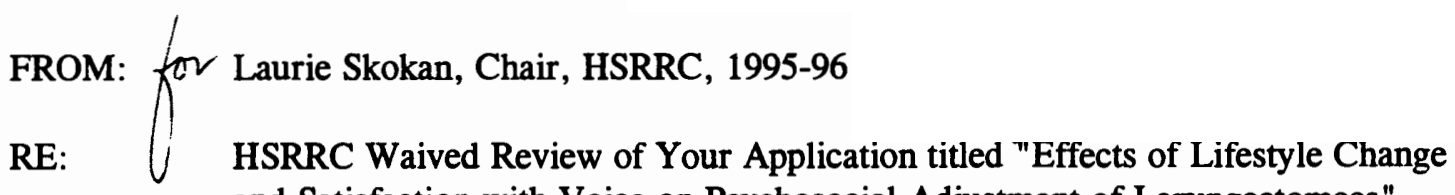
and Satisfaction with Voice on Psychosocial Adjustment of Laryngectomees"

Your proposal is exempt from further HSRRC review, and you may proceed with the study.

Even with the exemption above, it was necessary by University policy for you to notify this Committee of the Proposed research and we appreciate your timely attention to this matter. If you make changes in your research protocol, the Committee must be notified. This approval is valid for one year from date of issue.

Maureen Orr Eldred
Lisa Letcher-Glembo, Project Advisor 


\section{OFFICE OF GRADUATE STUDIES AND RESEARCH}

Research and Sponsored Projects

DATE: October 26, 1996

TO: $\quad$ Susan Nicks

FROM: for Vikki Vandiver, Chair, HSRRC 1996-97

RE: $\quad$ HSRRC approval of changes to your project, "Effects of Lifestyle Change and Satisfaction with Voice on Psychosocial Adjustment of Laryngectomees"

In response to your request for an approval of change in your original HSRRC application, the Human Subjects Research Review Committee has filed your addendum in your original application file. We note that there are no major alterations in the research plan originally proposed.

The Committee is satisfied that your provisions for protecting the rights and welfare of all subjects participating in the research are adequate. We would like to thank you for notifying the committee of your change, and ask that you document this as your approval for your applicaton. 
APPENDIX D

STUDY PROTOCOL INSTRUCTIONS 
Instructions for Filling Out Questionnaires

First, find a quiet space and time so that you can focus on the questionnaires. You can use either a pen or pencil.

\section{Written Consent Form}

Fill in your name at the top and on the signature line. Include the date at the bottom. This form will be separated from the questionnaires to ensure anonymity. It is the only paper that will have your name on it.

\section{Demographic questionnaire}

Here I am asking for your age, gender, when you had surgery, white type of speech you use, whether you belong to New Voice Club, and how your general health is these days. You are welcome to write any comments if you like.

\section{Lifestyle Questionnaire}

Part A is about your smoking and drinking habits before you were diagnosed with cancer. I need to find out whether you smoked and drank, and how much. The part about units of alcohol is probably the most challenging. Here are some examples:

1. You drank a glass of wine five times a week: a glass of wine is usually about 4 ounces. Under "Wine" the equivalent to one glass/4 ounces is " 1 unit." That ends up being 5 units a week. When you divide that by seven days $\mathrm{n}$ a week, you get less than one, so put a check next to the line that says "up to one unit per day."

2. You drank two beers almost every day: a bottle of beer is usually about 12 ounces. Two beers would be 24 ounces per day. Under "Beer," the equivalent is 8 ounces per unit. 24 ounces divided by 8 ounces would be 3 units. Make a check to the line that says 3 to 4 units per day.

3. You drank a beer every day during the week, and about four mixed drinks on weekends. A beer is 1.5 units, and 1.5 units times 5 days in a week is 7.5 units. Mixed drinks usually contain one shot each, so four mixed drinks is 4 units. 7.5 units during the week plus 4 units on weekends equals 11.5 units total for the week. Divide by 7 to find the average amount per day, and you get 1.6 units. Put a check mark next to the line that says 1 to 2 units per day.

If you don't feel like doing all that math, it is okay to estimate! Or feel free to call me and I will help you figure it out over the phone 
Part B of the lifestyle questionnaire asks about your current lifestyle. Do you currently smoke and drink, and if so, how much? Use the same measurements and way of figuring that was used above.

Your comments are welcome.

\section{Satisfaction with Voice Ouestionnaire}

The first question asks for your impressions about how well you are communicating with your alaryngeal voice and/or electrolarynx.

The second question asks for your opinion about whether and how speech therapy helped you in your adjustment following surgery.

The third question asks you to rate yourself. Is your alaryngeal speech understood all the time? Most of the time? Half the time? Hardly ever? Rating yourself with a "1" means you feel you are an excellent speaker and are always understood. A "5" means you are understood about half the time, and a "10" means you are hardly ever understood.

As usual, your comments about speech or communication are welcome.

\section{PAIS Questionnaire}

Please don't put your name on it!

This is a questionnaire about how cancer affected many areas of your life. The instructions on the cover say "effects that your recent illness has had on you." I realize that many of you are long-term survivors, and your illness was not all that recent. However, the questionnaire is still valid. The time period that we are interested in finding out about is the past $\mathbf{3 0}$ days. If there are some questions that don't apply to you, it is okay to leave them blank. Do answer all the questions that apply to you. Try to find the sentence that most closely matches your experience.

If you need some help with the questionnaires, or are unsure how to answer questions, feel free to give me a call.

Your participation in this study is greatly appreciated

\section{Susan Nicks}

4107 S.E. Washington Street

Portland, OR 97214

(503) 236-1651

e-mail: atsuonicki@cenornet.com 
APPENDIX E

DEMOGRAPHIC QUESTIONNAIRE 
Demographic Questionnaire

Subject \#

Date

Please complete the following questions:

Your Age:

Circle one: Male Female

Month and year of laryngectomy surgery:

What type of speech do you use? (Circle all that apply)

Esophageal Tracheoesophageal Artificial Larynx None of the Above

Are you a member of the New Voice Club? YES NO

How would you describe your general health at this time?

Excellent Very Good Good Fair Poor 


\section{APPENDIX F}

\section{LIFESTYLE QUESTIONNAIRE}


Lifestyle Questionnaire

Subject \#

Date

\section{Part A}

Please complete the following questions. This part of the questionnaire asks about your smoking and drinking habits before your cancer diagnosis.

Have you ever smoked? YES NO

How many years did you smoke?

How much did you smoke per day before your cancer diagnosis? up to $1 / 2$ pack per day

$1 / 2$ pack to 1 pack per day

1 to 2 packs per day more than 2 packs per day I smoked cigars (How many per day? I smoked pipes (How many per day?

Before your cancer diagnosis, did you ever drink alcohol? YES NO

If yes, check the answer that describes your typical alcohol intake before your cancer diagnosis. drank 1-2 times per year drank 1-2 times per month drank 1-2 times per week drank daily other (please describe

If you drank more than 1-2 times per week before your cancer diagnosis, what is the average amount you consumed per day?

Please use the appropriate measurement:

For hard liquor, 1 unit $=1$ shot whiskey

For wine, 1 unit $=4 \mathrm{oz}$. wine

For beer, 1 unit $=8 \mathrm{oz}$. beer

up to 1 unit per day

1 to 2 units per day

2 to 3 units per day

3 to 4 units per day

4 or more units per day 


\section{Part B}

This part of the questionnaire asks about your smoking and drinking habits after your laryngectomy surgery.

Did you stop smoking after your laryngectomy surgery? YES NO

Do you currently smoke? YES NO

If you currently smoke, how much do you smoke? up to $1 / 2$ pack per day $1 / 2$ pack to 1 pack per day 1 to 2 packs per day more than 2 packs per day I smoked cigars (How many per day? I smoked pipes (How many per day?

Did you stop drinking alcohol, or reduce the amount you drank after your surgery?

\section{STOPPED REDUCED NEITHER}

Do you currently drink any alcohol? YES NO

If yes, check the answer that describes your typical alcohol intake since your laryngectomy surgery.

drink 1-2 times per year drink 1-2 times per month drink 1-2 times per week drink daily other (please describe

If you currently drink more than 1-2 times per week, what is the average amount you consume per day?

up to 1 unit per day

1 to 2 units per day

2 to 3 units per day

3 to 4 units per day

4 or more units per day

Comments 


\section{APPENDIX G}

SATISFACTION WITH VOICE SCALE 


\section{Satisfaction with Voice Scale}

Subject \#

Date

1) Describe how you think you are communicating.

2) How did speech therapy help with adjusting to or coping with cancer?

3) On a scale of 1 to 10 , how would you rate your ability to be understood?

$\begin{array}{lccccccccc}1 & 2 & 3 & 4 & 5 & 6 & 7 & 8 & 9 & \begin{array}{l}10 \\ \text { Rarely } \\ \text { Understood }\end{array} \\ \begin{array}{l}\text { Understood } \\ \text { all the Time }\end{array} & & & & & & & & \end{array}$

4) Additional comments, if any: 